\title{
Acquisition of cellular properties during alveolar formation requires differential activity and distribution of mitochondria
}

Kuan Zhang ${ }^{1}$, Erica Yao ${ }^{1}$, Julia Wong ${ }^{1}$, Paul J. Wolters ${ }^{2}$ and Pao-Tien Chuang $1 \S$

1 Cardiovascular Research Institute, University of California, San Francisco, CA 94158, USA

2 Division of Pulmonary, Critical Care, Allergy and Sleep Medicine, Department of Medicine, University of California, San Francisco, CA, USA

§Correspondence and requests for materials should be addressed to Pao-Tien Chuang (email: pao-tien.chuang@ucsf.edu).

Running Title: Mitochondria and alveologenesis 


\begin{abstract}
Alveolar formation requires coordinated movement and interaction between alveolar epithelial cells, mesenchymal myofibroblasts and endothelial cells/pericytes to produce secondary septa. These processes rely on the acquisition of distinct cellular properties to enable ligand secretion for cell-cell signaling and initiate morphogenesis through cell migration and cell shape change. In this study, we showed that mitochondrial activity and distribution play a key role in bestowing cellular functions on both alveolar epithelial cells and mesenchymal myofibroblasts for generating secondary septa to form alveoli. These results suggest that mitochondrial function is tightly regulated to empower cellular machineries in a spatially specific manner. Indeed, such regulation via mitochondria is required for secretion of platelet-derived growth factor from alveolar epithelial cells to influence myofibroblast proliferation and migration. Moreover, mitochondrial function enables myofibroblast migration during alveolar formation. Together, these findings yield novel mechanistic insights into how mitochondria regulate pivotal steps of alveologenesis. They highlight selective utilization of energy and diverse energy demands in different cellular processes during development. Our work serves as a paradigm for studying how mitochondria control tissue patterning.
\end{abstract}




\section{Introduction}

Production of alveoli during development and following lung injury is essential for lung function (1-3). Defective alveologenesis underlies bronchopulmonary dysplasia (BPD) (4) and ongoing destruction of alveoli is characteristic of chronic obstructive lung disease (COPD) (5). COPD is a major cause of morbidity and mortality globally $(6,7)$. During alveolar formation, alveolar epithelial cells (type I [AT1] and type II [AT2] cells), myofibroblasts and endothelial cells/pericytes undergo coordinated morphogenetic movement to generate secondary septa within saccules. As a result, secondary septa comprise of a layer of alveolar epithelial cells that ensheathes a core of myofibroblasts and endothelial cells/pericytes. Secondary septa formation (or secondary septation) is the most important step during alveolar formation. Platelet-derived growth factor (PDGF) produced by alveolar epithelial cells is a key player in controlling myofibroblast proliferation and migration during alveologenesis $(8,9)$. In response to PDGF signaling, myofibroblasts migrate to the prospective site of secondary septation and secrete elastin. Myofibroblasts and endothelial cells/pericytes are subsequently incorporated with alveolar epithelial cells to form secondary septa. All of these principal components play a key role in driving secondary septa formation (2). Generation of alveoli increases the surface area and efficiency of gas exchange, enabling high activity in terrestrial environments. Despite the progress that has been made, our mechanistic understanding of alveologenesis remains incomplete.

Mitochondrial activity is essential for every biological process and mitochondria provide a major source of ATP production through oxidative phosphorylation (OXPHOS) $(10,11)$. Unexpectedly, we have limited mechanistic insight into how mitochondria control cellular processes in vivo. In this regard, little is known about if and how mitochondria regulate alveolar 
formation. Many genetic and molecular tools have been developed in mice to study

mitochondrial function. They offer a unique opportunity to address the central question of how mitochondria control alveologenesis at the molecular level.

Mitochondria exhibit dynamic distribution within individual cells. This process is mediated by the cytoskeletal elements that include microtubules, F-acitn and intermediate filaments. For instance, Mirol (Mitochondrial Rho GTPase 1), which is also called Rhot1 (ras homolog family member 1), encodes an atypical Ras GTPase and plays an essential role in mitochondrial transport (12). Miro1 associates with the Milton adaptor (TRAK1/2) and motor proteins (kinesin and dynein), and tethers the adaptor/motor complex to mitochondria. This machinery facilitates transport of mitochondria via microtubules within mammalian cells. Whether regulated mitochondrial distribution is essential for lung cell function during alveologenesis is unknown.

In this study, we have demonstrated a central role of mitochondrial activity and distribution in conferring cellular properties to alveolar epithelial cells and myofibroblasts during alveolar formation. In particular, PDGF ligand secretion from alveolar epithelial cells and motility of myofibroblasts depend on regulated activity and distribution of mitochondria. Moreover, loss of mitochondrial function does not have a uniform effect on cellular processes, indicating diverse energy demands in vivo. We also reveal regulation of mitochondrial function by the mTOR complex $1(13,14)$ during alveolar formation and establish a connection between mitochondria and COPD/emphysema. Taken together, these findings provide new insight into how different cell types meet unique energy demands to engender distinct cellular properties during alveolar formation. 


\section{Results}

\section{Mitochondria display dynamic subcellular distribution in alveolar epithelial cells and mesenchymal myofibroblasts during alveolar formation}

To uncover the functional role of mitochondria during alveologenesis, we first examined the distribution of mitochondria in murine lung cells involved in alveolar formation. We used antibodies against mitochondrial components to visualize the distribution of mitochondria in lung epithelial cells and myofibroblasts. For instance, we performed immunostaining on lung sections derived from Sox $9^{C r e /+}$; ROSA26 $6^{m T m G /+}$ mice with anti-MPC1 (mitochondrial pyruvate carrier 1) and anti-MTCO1 (mitochondrially encoded cytochrome C oxidase I) (15). In particular, anti-MPC1 serves as a general marker for mitochondria. Lung epithelial cells were labeled by GFP produced from the $R O S A 26^{m T m G}$ reporter (16) due to selective Cre expression in SOX9+ epithelial cells (17). We found that mitochondria were widely distributed in alveolar epithelial cells (distinguished by T1 $\alpha$ and SPC for AT1 and AT2 cells, respectively) and myofibroblasts (marked by PDGFRA, PDGF receptor a, and smooth muscle actin, SMA) (18, 19) (Figure 1A). This is consistent with an essential role of mitochondrial activity in proper functioning of lung cells. In addition, we observed an uneven subcellular distribution of mitochondria (Figure 1A, 1B). For instance, mitochondria were concentrated in areas adjacent to the trans-Golgi network $\left(\mathrm{TGN} 8^{+}\right)$in alveolar epithelial cells where proteins were sorted to reach their destinations through vesicles and in areas that surrounded SMA in myofibroblasts (Figure 1A, 1B). This finding suggests that localized mitochondrial distribution is required for cellular functions in mammalian lungs.

\section{Postnatal removal of mitochondrial activity in the lung leads to defective alveologenesis}


We first tested if mitochondrial activity is required for alveologenesis by inactivating Tfam (transcription factor A, mitochondria), which encodes a master regulator of mitochondrial transcription (20), in the mouse lung after birth. We produced $C A G G^{C r e E R /+}$; ROSA26 ${ }^{m T m G /+}$ (control) and Tfamflf; $C A G G^{C r e E R /+} ; R O S A 26^{m T m G /+}$ mice. Tamoxifen was administered to neonatal mice to activate CreER and lungs were collected at postnatal (P) day 10 (Figure 2A). CreER expression under the $C A G$ promoter/enhancer (21) was ubiquitous in lung cells, including NKX2.1+ epithelial cells and PDGFRA ${ }^{+}$fibroblasts/myofibroblasts, and converted a floxed allele of Tfam (Tfamf) (22) into a null allele (Figure 2B). We noticed that multiple regions in the lungs of mutant mice displayed alveolar defects concomitant with an increased mean linear intercept (MLI), a measure of air space size $(23,24)$ (Figure 2C, 2D). Alveolar defects were associated with disorganized SMA (Figure 2E). We anticipated that Tfam removal led to shutdown of mitochondrial transcription and loss of mitochondrial activity. Indeed, the relative ratio of mitochondrial DNA (mtDNA) (25), 16S rRNA and mtND1 (mitochondrially encoded NADH dehydrogenase 1), to nuclear DNA (nDNA), Hk2 (hexokinase 2), was reduced in Tfamdeficient lungs compared to controls (Figure 2F). Loss of Tfam was accompanied by diminished immunoreactivity of MTCO1, the expression of which was controlled by Tfam. (Figure 2G). Together, these results indicate that mitochondrial activity is required for alveolar formation.

\section{Selective loss of mitochondrial activity in the lung epithelium disrupts alveologenesis}

To investigate the function of mitochondrial activity in distinct compartments, we selectively eliminated mitochondrial activity in either the lung epithelium or mesenchyme. We produced control and Tfamflf; Sox9Cre/+ mice to establish a platform for mechanistic studies on mitochondrial activity in lung epithelial cells during alveolar formation. The Sox9Cre mouse line 
(17) is highly efficient in removing sequences flanked by loxP sites in the distal lung epithelium. Sox9-Cre is active at or later than 11.5 days post coitus $(\mathrm{dpc})$ and converted Tfamf into a null allele and disrupted mitochondrial activity (Figure 3, figure supplement 1A). Tfamflf; Sox9Cre/+ mice were born at the expected Mendelian frequency and could not be distinguished from their wild-type littermates by their outer appearance or activity at birth. Moreover, histological analysis revealed no difference between control and mutant lungs prior to P5, confirming that branching morphogenesis and saccule formation were unaffected by inactivating Tfam in SOX9+ cells (Figure 3, figure supplement 2A). In addition, proliferation and differentiation of alveolar type I and type II cells proceeded normally in Tfam flf; Sox9Cre/+ lungs (Figure 3, figure supplement 2B). These results highlight a difference in dependence on mitochondrial activity in distinct cellular processes during development. To uncover the cellular processes that are highly dependent on mitochondrial activity, we investigated alveolar formation in control and Tfamflf; Sox9Cre/+ lungs.

After P5, Tfamflf; Sox9Cre/+ mice could be discerned by their slightly reduced body size in comparison with the littermate controls. Histological analysis of Tfamflf; Sox $9 \mathrm{Cre} /+$ lungs at various postnatal stages revealed defects in secondary septa formation with an increased MLI (Figure 3A, 3B). Most Tfamflf; Sox9Cre/+ mice survived beyond P30, permitting analysis of the progression of their alveolar defects. These results reveal a critical role of mitochondrial activity in lung epithelial cells during alveologenesis.

\section{Disruption of mitochondrial distribution in the lung epithelium disturbs alveologenesis}

As described above, mitochondria display dynamic distribution in lung cells, raising the possibility that proper subcellular distribution of mitochondria is vital for cellular function during 
alveolar formation. To test this hypothesis, we generated control and Mirolfff; Sox 9 Crel+ mice. Sox9Cre converted a floxed allele of Mirol (Mirof) (26) to a null allele in SOX9+ alveolar epithelial cells. Loss of Mirol is expected to perturb normal subcellular distribution of mitochondria. Mirolflf; Sox 9 Cre/+ mice were born at the expected Mendelian frequency and cannot be distinguished from their wild-type littermates by their outer appearance or activity at birth. Similarly, no difference between control and mutant lungs prior to P5 was detected by histological analysis (Figure 3, figure supplement 2C, 2D). After P5, Mirolfff; Sox9Crel+ mice displayed defects in secondary septation with an increased MLI. The alveolar phenotypes could first appear anywhere between P5 and P12 (Figure 3C, 3D). These results support the notion that localized mitochondrial distribution plays a functional role in alveolar formation. We noticed that the alveolar defects in Mirolfff; Sox9Cre/+ lungs were less severe than those in Tfamflf; Sox $9 \mathrm{Cre} /+$ lungs. This is likely due to the fact that only the distribution and not the activity of mitochondria was perturbed in Mirolfff; Sox9Crel+ lungs.

\section{PDGF signal reception is perturbed and the number of mesenchymal myofibroblasts is} reduced in the absence of proper mitochondrial activity or distribution in the lung

\section{epithelium}

We examined various lung cell types in Tfamflf; Sox9Cre/+ lungs to explore the molecular basis of their alveolar phenotypes. Interestingly, the number of myofibroblasts marked by PDGFRA was reduced in the absence of epithelial Tfam (Figure 4A, 4D). Likewise, we found that the number of myofibroblasts was reduced in Mirolfff; Sox9Crel+ lungs where epithelial Mirol was lost (Figure 4G, 4I). A diminished population of myofibroblasts in Tfam ${ }^{f / f}$; Sox9Crel+ and Mirolfff; Sox9Cre/+ lungs prompted us to investigate whether PDGF signaling was disrupted. 
Phosphorylation of PDGFRA (p-PDGFRA), indicative of PDGF signaling, was reduced in Tfamflf; Sox9Crel+ or Mirolfff; Sox9Crel+ lungs (Figure 4A, 4G). This observation suggests that PDGF signal reception by myofibroblasts was impaired in Tfamfff; Sox9Cre/+ or Mirolff; Sox9Cre/+ lungs. Defective PDGF signal reception in myofibroblasts could be due to lack of PDGF production, trafficking or release.

We found that production of the PDGF ligand (PDGFA) in alveolar epithelial cells was unaffected in Tfamflf; Sox9Cre/+ or Mirolfff; Sox9Cre/+ lungs by qPCR analysis (Figure 4E, 4J). To substantiate this model, we utilized a PDGF reporter mouse line (Pdgfaex4COIN) (27) that faithfully recapitulates the spatial and temporal expression of Pdgfa. Of note, no reliable PDGF antibody is available to detect PDGF in lungs or other tissues $(19,27)$. We generated Pdgfa ex4COIN/+ ; Sox9Cre/+ (control) and Tfamflf; Pdgfaex4COIN/+ ; Sox9Cre/+ mice. Cre recombinase activated $\beta$-galactosidase (lacZ) expression in Pdgfa-expressing cells from the Pdgfa ex4COIN allele. We found that $L a c Z$ expression in $P d g f a$-expressing cells (i.e., alveolar epithelial cells) displayed a similar pattern and intensity between control and Tfam-deficient lungs (Figure 4C). Together, these results pointed to disrupted PDGF trafficking or release. This defect would subsequently disturb signal reception in mesenchymal myofibroblasts of Tfamflf; Sox 9 Cre/+ and Mirolflf; Sox9Crel+ lungs.

\section{PDGF secretion from lung cells is diminished without proper mitochondrial activity or}

\section{distribution}

Our model posits that secretion of PDGF ligand from Tfam- and Mirol-deficient alveolar epithelial cells is compromised. To test this idea, we derived Tfam-and Mirol-deficient cells from Tfamflf; PdgfraCrel+ and Mirolflf; PdgfraCre/+ lungs (see below), respectively. We 
transduced control and Tfam- and Mirol-deficient cells with lentiviruses that produced epitopetagged PDGF. Using this assay, we determined the amount of PDGF released from control and Tfam- and Mirol-deficient cells (Figure 4F, 4K). PDGF levels in the conditioned media derived from Tfam- or Mirol-deficient cells were reduced compared to controls (Figure 4F, 4K). These findings support a model in which loss of mitochondrial activity or distribution results in a failure of vesicular transport and PDGF release from alveolar epithelial cells. We surmise that these defects are in part due to an incapacitated actomyosin cytoskeleton caused by reduced mitochondrial function.

\section{Selective loss of mitochondrial activity or distribution in myofibroblasts compromises}

\section{alveologenesis}

We then investigated the functional requirement of mitochondria in the lung mesenchyme. To this end, we produced control and Tfamflf; PdgfraCre/+ (28) mice for Tfam inactivation in lung fibroblasts/myofibroblasts. Cre expression in $\mathrm{PDGFRA}^{+}$myofibroblasts eliminated Tfam and mitochondrial activity. Indeed, the expression of MTCO1, a transcriptional target of Tfam, was reduced in lung fibroblasts/myofibroblasts derived from Tfam ${ }^{f l f} ; \mathrm{PdgfraCrel+}$ mice (Figure 3, figure supplement 1B). Prior to P3, Tfamflf; PdgfraCre/+ mice could not be distinguished from their wild-type littermates by appearance, activity, morphological and immunohistochemical analysis (Figure 5, figure supplemental 1A, 1B). This suggests that loss of Tfam in the lung mesenchyme did not affect branching morphogenesis or saccule formation (29). This permitted us to assess the contribution of mesenchymal mitochondria to alveolar formation. Histological analysis of Tfam flf; PdgfraCrel+ lungs at various postnatal stages prior to P5 revealed defective 
secondary septa formation with an increased MLI (Figure 5A, 5B). Most Tfamflf; PdgfraCre/+ mice died before P30.

We also generated control and Tfamflf; Dermol Crel+ mice, in which Tfam was eliminated by mesenchymal Dermol Cre (30). Tfamfff; DermolCre/+ mice exhibited alveolar defects (Figure 5, figure supplemental 2A, 2B) similar to those in Tfamflf; PdgfraCre/+ mice, further supporting a central role of mitochondrial activity in the lung mesenchyme during alveologenesis.

Of note, we bred Lrpprcfff; PdgfraCre/+ mice as an alternative means to disrupt mitochondrial activity. Lrpprc (Leucine-rich PPR motif-containing) (31) is required for mitochondrial translation. Similarly, PdgfraCre converted a floxed allele of Lrpprc (Lrpprcf) into a null allele. Lrpprc affects different aspects of mitochondrial activity and serves the purpose of confirming our findings using Tfam. We found that Lrpprcfff; PdgfraCre/+ mice developed alveolar defects (Figure 5, figure supplemental 2C, 2D), albeit the phenotypes were less severe than those in Tfamflf; PdgfraCre/+ mice. This was likely due to the presence of residual proteins after removal of Lrpprc. Together, these studies establish a crucial role of mitochondrial activity in myofibroblasts for alveologenesis.

We went on to determine whether proper subcellular distribution of mitochondria in myofibroblasts is necessary for their function during alveolar formation. We generated control and Mirolfff; PdgfraCre/+ mice (Figure 5, figure supplemental 1C, 1D). In this case, the subcellular distribution of mitochondria is expected to be perturbed in mesenchymal myofibroblasts as indicated by loss of proper MTCO1 distribution in myofibroblasts derived from lungs of Mirolflf; PdgfraCre/+ mice (Figure 3, figure supplement 1C). Mirolflf; PdgfraCre/+ mice exhibited alveolar defects, milder than those in Tfamflf; Pdgfra ${ }^{C r e l+}$ lungs (Figure 5C, 5D). 
We discovered that myofibroblast proliferation was reduced in Tfamflf; PdgfraCre/+ or Mirolfff; PdgfraCre/+ lungs compared to controls (Figure 6A, 6B, 6E, 6F), suggesting defective PDGF signal reception. This may be related to a failure in PDGFR trafficking when mitochondrial function is impaired. As a result, the pool of PDGFRA ${ }^{+}$myofibroblasts was decreased with a concomitant reduction in SMA (encoded by Acta2) and/or elastin (Figure 6C, $6 \mathrm{G})$, contributing to alveolar defects. Taken together, these findings suggest that proper activity and distribution of mitochondria in mesenchymal myofibroblasts are critical to generating a sufficient number of myofibroblasts for secondary septation.

\section{Migration of myofibroblasts is reduced without proper mitochondrial activity or distribution, which is associated with a disrupted cytoskeleton}

We speculate that myofibroblasts deficient in Tfam are also defective in their ability to migrate to the prospective site of secondary septation. To test this idea, we isolated myofibroblasts from control and Tfam flf; PdgfraCre/+ lungs and seeded them onto the migration chamber for wound healing assays (32). The rate of myofibroblast migration into the cell-free area was measured. While control myofibroblasts occupied the cell-free area after 36-48 hr, only scant Tfamdeficient myofibroblasts were detected in the cell-free area (Figure 6D). This result indicates that mobility of myofibroblasts was compromised due to loss of mitochondrial activity in these cells. We conjecture that the migration defect was in part due to an incapacitated actomyosin cytoskeleton without mitochondrial activity. Consistent with this idea, organization of the cytoskeleton (labeled by phalloidin) was perturbed in Tfamflf; PdgfraCre/+ lungs in comparison with controls (Figure 6A). 
Similarly, myofibroblasts derived from Mirolfff; PdgfraCre/+ lungs displayed a compromised response in wound healing assays compared to controls (Figure 6H). Together, these results affirm the role of mitochondrial activity and distribution in regulating myofibroblast migration during alveologenesis.

\section{The mTOR complex 1 regulates mitochondrial function and alveologenesis}

We have discovered an essential role of mitochondria in controlling alveolar formation. To dissect the signaling cascade that regulates mitochondrial function during alveologenesis, we first investigated mTOR complex 1 (mTORC1), a known regulator of mitochondrial activity and biogenesis. We generated control, Rptorflf; Sox9Cre/+ and Rptorflf; PdgfraCrel+ mice. Rptor (Raptor, rapamycin-sensitive regulatory associated protein of mTOR) (33) encodes an essential component of mTORC1, which includes RPTOR, mTOR and several other proteins. Rptorff; Sox9Cre/+ and Rptorflf; PdgfraCrel+ mice failed to survive to term, precluding the analysis of potential alveolar phenotypes.

To circumvent this problem, we produced control and Rptorfff; $C A G G^{C r e E R /+}$ mice and administered tamoxifen postnatally (Figure 7A). Analysis of Rptorfff; CAGGCreER/+ mice at P10 revealed alveolar defects with an increased MLI (Figure 7B, 7C). Defective secondary septation in Rptorfff; CAGGCreER/+ lungs was associated with reduced SMA (Figure 7D). As expected, the protein levels of phosphorylated ribosomal protein S6 (p-RPS6), a downstream target of mTORC1, was decreased in lysates from Rptor-deficient lungs compared to controls (Figure 7E). The relative ratio of mtDNA to nDNA was reduced in Rptor-deficient lungs (Figure 7F). Moreover, immunoreactivity of both MPC1 and MTCO1 was diminished in Rptorflf; $C A G G^{C r e E R /+}$ lungs compared to controls (Figure 7G). These findings show that elimination of 
Rptor in the lung resulted in loss of mitochondria. Impaired mitochondrial function then contributed to alveolar defects. Together, our results suggest that mTORC1 controls alveolar formation partly through its effects on mitochondrial function.

\section{Mitochondrial copy number and TFAM protein levels are decreased in lungs from COPD/emphysema patients}

To explore whether studies of mitochondrial function in alveolar formation in mice can shed new light on human diseases, we assessed the status of mitochondria in lung tissues of normal subjects and COPD/emphysema patients (Figure 8A). We found that the copy number of mitochondria (25) relative to nuclear DNA was significantly reduced in COPD/emphysema patients (Figure 8B). In addition, TFAM protein levels were reduced in lysates from emphysema lungs compared to normal lungs (Figure 8C). These results established a connection between mitochondrial function and pathogenesis of COPD/emphysema. Interestingly, a disorganized cytoskeleton was noted in COPD/emphysema lungs, in which actin bundles seen in normal alveoli were sparse (Figure 8D). We did not detect a difference in the protein levels of either ribosomal protein S6 (RPS6) or phosphorylated RPS6 (p-RPS6), a downstream target of mTORC1, in lysates from emphysema and normal lungs. However, we observed heterogeneous expression of p-RPS6 in emphysema lungs (Figure 8E). Whether regional reduction of p-RPS6 is correlated with disease progression needs future studies. Taken together, these findings complement our mouse work and lay the foundation for further investigation into the disease mechanisms of COPD/emphysema. 


\section{Discussion}

Our studies have provided new insight into how mitochondrial function controls alveolar formation. We discovered a major role of mitochondria in conferring requisite cellular properties to both alveolar epithelial cells and myofibroblasts during alveologenesis. These findings define the molecular basis of the functional requirement of mitochondria in distinct compartments. They also add a new layer of complexity to the interactions between the major players of secondary septa. Moreover, our work establishes the foundation for investigating the interplay between mitochondria and signaling pathways in endowing cellular properties in alveologenesis during development and following injury. We expect that this framework will be applicable to other developmental systems (Figure 9).

Removal of mitochondria either globally or in distinct compartments in the lung results in alveolar defects. We found that not all cellular processes are perturbed to the same extent when mitochondrial activity or distribution is perturbed. For instance, while alveolar development is disrupted, saccule formation and cell type specification are unaffected. These observations highlight the differential requirement of mitochondrial function in a given cellular process. Events that necessitate a higher demand of energy will be the first to exhibit phenotypes once mitochondrial function is compromised. It implies that the severity of phenotypes resulting from a reduction in mitochondrial function could be used to characterize the energy demand of a particular cellular process in development. This information cannot be obtained from cell-based studies.

Our work has focused on PDGF ligand secretion. While other pathways have not been interrogated, different pathways may exhibit a varying degree of dependence on mitochondrial function. Similarly, this could provide a new way to functionally categorize signaling pathways 
on the basis of their energy demand in vivo. Such insight will provide a new blueprint of how cells dispense its energy source in tissues.

Secretion of the PDGF ligand from alveolar epithelial cells is impaired due to mitochondrial dysfunction in either activity or distribution. We surmise that the actomyosin cytoskeleton likely underlies this defect. However, it is also possible that the energy produced by mitochondria is required in many key steps of vesicular transport and membrane fusion. Additional insight would come from a careful assessment of the dependence of each process on mitochondrial function. Similarly, failure to power the actomyosin cytoskeleton in myofibroblasts due to disruption of mitochondrial activity or distribution could cause the migratory defect. ATP produced by mitochondria is known for the assembly of the actomyosin cytoskeleton. By contrast, how regulation of mitochondrial distribution is superimposed upon the formation of the actomyosin cytoskeleton is less understood at the molecular level. This scenario is further complicated by the observation that F-actin and intermediate filaments also play a key role in mitochondrial dynamics and functions (34). Technical advances that enable live imaging (35) of mitochondria (36) and the cytoskeleton would provide new tools to address this important issue.

We have uncovered the role of mitochondria in alveolar epithelial cells and myofibroblasts during alveolar formation. Whether the function of endothelial cells/pericytes also relies on mitochondrial activity and/or distribution in this process is unknown $(37,38)$. This would require future studies using an approach similar to that employed in this work. Again, the dependence of cell types, cellular processes and signaling pathways on mitochondrial function can only be revealed through studies in vivo. 
We envision that a complex regulatory network must be in place to regulate mitochondrial number and distribution in distinct cellular processes. Our work shows that mTOR complex 1 is a key player in controlling mitochondrial function during alveolar formation. This is consistent with the role of mTORC1 in regulating mitochondrial function in cell-based assays. Identifying additional components in the signaling network would reveal the key hubs in the signaling network that control mitochondrial function during alveologenesis.

Mitochondrial copy number and TFAM expression levels are reduced in the lungs of COPD/emphysema patients. However, it is unclear if mitochondrial activity and distribution contribute to the pathogenesis of COPD/emphysema. This would rely on the analysis of lungs at different stages of disease progression. It also necessitates new approaches that enable a mechanistic correlation between mitochondrial function and disease progression. For instance, analysis of the transcriptome and proteome of various lung cell types at single cell levels could reveal alterations in a subset of cells that herald the process of emphysematous changes.

Taken together, our work has yielded new molecular insight into the old question of energy utilization in vivo. In particular, energy production by mitochondria is channeled in a spatially specific manner to enable cellular processes in distinct cell types. Diverse cell types and cellular processes have a unique energy demand, which is likely executed by a signaling network. These investigations form the basis of additional studies to explore this new concept in alveolar formation and repair and in other processes in vivo. 


\section{Materials and methods}

\section{Animal husbandry}

Mouse strains used in this study are listed in the Key Resources table. All mouse experiments described in this study were performed according to the protocol approved by the Institutional Animal Care and Use Committee (IACUC) of the University of California, San Francisco (UCSF).

\section{Tamoxifen administration}

Tamoxifen was prepared by dissolving in corn oil to a concentration of $50 \mathrm{mg} / \mathrm{ml}$ (32). For postnatal $(\mathrm{P})$ injection, the tamoxifen stock was diluted $1 / 10$ in corn oil to make a final concentration of $5 \mathrm{mg} / \mathrm{ml} .50 \mu \mathrm{L}$ of tamoxifen was delivered through oral gavage or direct injection into the stomach of neonatal mice.

\section{Measurement of mean linear intercept (MLI)}

Measurement of MLI was performed as previously reported (32). Briefly, 15 fields without visible blood vessels or airways from three different histological sections per animal were captured at 20x magnification using a Nikon Eclipse E1000 microscope. A grid with horizontal and vertical lines (10 each) was superimposed on the images using ImageJ. The mean linear intercept $(\mathrm{Lm})$ was calculated as: $\mathrm{Lm}=\mathrm{L} / \mathrm{N}$, where $\mathrm{L}$ is the total length of horizontal plus vertical lines, and $\mathrm{N}$ is the total number of the intercepts.

\section{Whole lung imaging, histology and immunohistochemistry}


To image the whole lungs that carried GFP or RFP, dissected mouse lungs at the indicated stages were placed under a Nikon Eclipse E1000 microscope equipped with a SPOT 2.3 CCD camera.

Mouse lungs at indicated time points were collected and fixed with $4 \%$ paraformaldehyde (PFA) in PBS on ice for $1 \mathrm{hr}$. The tissues were embedded in paraffin wax or OCT, and sectioned at $7 \mu \mathrm{m}$. For histological analysis of lung sections, hematoxylin and eosin (H\&E) staining was performed as previously described $(32,39)$. Images were taken using a SPOT 2.3 CCD camera connected to a Nikon Eclipse E1000 microscope.

To detect Pdgfa in lung cells, lungs from Sox $9 \mathrm{Cre}^{++} ; \mathrm{Pdgfa}^{\text {ex4COIN/+ }}$ (control) and Tfamflf;

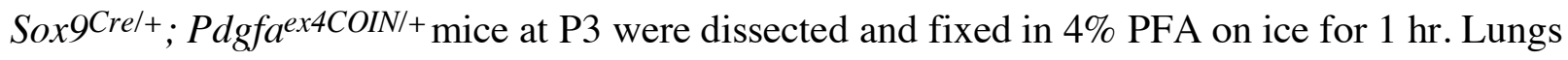
were washed in $0.02 \%$ NP40 in PBS for $2 \mathrm{hr}$, then placed in X-gal staining solution (5 mM $\mathrm{K}_{3} \mathrm{Fe}(\mathrm{CN})_{6}, 5 \mathrm{mM} \mathrm{K}_{4} \mathrm{Fe}(\mathrm{CN})_{6}, 2 \mathrm{mM} \mathrm{MgCl}{ }_{2}, 0.01 \%$ sodium deoxycholate, $0.02 \% \mathrm{NP}-40,1$ $\mathrm{mg} / \mathrm{ml} \mathrm{X}$-gal) for $72 \mathrm{hr}$ at $37^{\circ} \mathrm{C}$. The stained lungs were paraffin embedded and sectioned. Images were taken using a SPOT 2.3 CCD camera connected to a Nikon Eclipse E1000 microscope.

Immunofluorescence was performed as previously described (39). Antibodies used in this study are listed in the Key Resources table. The primary antibodies used for wax sections were: chicken anti-GFP (1:200, abcam, Cat\# ab13970), rabbit anti-NKX2.1 (1:100, Epitomics, Cat\# 2044-1), goat anti-CC10 (1:200, Santa Cruz Biotechnology, Cat\# sc-9773), mouse antiacetylated tubulin (1:200, Sigma-Aldrich, Cat\# T6793), rabbit anti-prosurfactant protein C (proSP-C) (1:200, MilliporeSigma, Cat\# AB3786), hamster anti-T1 $\alpha$ (1:200, Developmental Studies Hybridoma Bank, Cat\# 8.1.1), mouse anti-HOPX (1:100, Santa Cruz Biotechnology, Cat\# sc-398703). The primary antibodies used for frozen sections were: rabbit anti-MPC1 (1:100, Millipore/Sigma, Cat\# HPA045119), rat anti-E-cadherin (1:200, Invitrogen, Cat\# 13- 
1900), mouse anti-MTCO1 (1:100, abcam, Cat\# ab14705), chicken anti-GFP (1:300, abcam, Cat\# ab13970), mouse anti-ACTA2 (1:200, Thermo Scientific Lab Vision, Cat\# MS-113-P0), rat anti-PECAM-1 (CD31) (1:150, Santa Cruz Biotechnology, Cat\# sc-18916), rabbit anti-PDGFRA (1:150, Cell Signaling Technology, Cat\# 3164), rabbit anti-phospho-PDGFRA (Tyr754) (1:100, Cell Signaling Technology, Cat\# 2992), mouse anti-S6 Ribosomal Protein (1:100, Cell Signaling Technology, Cat\# 2317), rabbit anti-Phospho-S6 Ribosomal Protein (Ser235/236) (1:100, Cell Signaling Technology, Cat\# 4856). Secondary antibodies and conjugates used were: donkey antirabbit Alexa Fluor 488 or 594 (1:1000, Life Technologies), donkey anti-chicken Alexa Fluor 488 or 647 (1:1000, Life Technologies), donkey anti-mouse Alexa Fluor 488 or 594 (1:1000, Life Technologies), and donkey anti-rat Alexa Fluor 594 (1:1000, Life Technologies). The biotinylated secondary antibodies used were: goat anti-hamster (1:1000, Jackson ImmunoResearch Laboratories), donkey anti-rabbit (1:1000, Jackson ImmunoResearch Laboratories), donkey anti-rat (1:1000, Jackson ImmunoResearch Laboratories) and horse antimouse (1:1000, Jackson ImmunoResearch Laboratories). The signal was detected using streptavidin-conjugated Alexa Fluor 488, 594, or 647 (1:1000, Life Technologies) or HRPconjugated streptavidin (1:1000, Perkin-Elmer) coupled with fluorogenic substrate Alexa Fluor 594 tyramide for 30 s (1:200, TSA kit; Perkin Elmer). F-actin was stained with rhodamineconjugated phalloidin (1:200, Sigma) in PBS for $2 \mathrm{hr}$. Since the filamentous actin is sensitive to methanol, ethanol and high temperature, we only used OCT-embedded frozen sections for Factin staining.

Confocal images were captured on a Leica SPE laser-scanning confocal microscope. Adjustment of red/green/blue/grey histograms and channel merges were performed using LAS AF Lite (Leica Microsystems). 


\section{Myofibroblast proliferation assays}

The rate of cell proliferation was determined through EdU incorporation as previously described (32). Mouse pups at the indicated time points were intraperitoneally injected with EdU/PBS solution for $1 \mathrm{hr}$ before dissection. The Click-iT EdU Alexa Fluor 488 Imaging Kit (Life Technologies) was used to quantify EdU incorporation. The sections were co-stained with antibody against PDGFRA. Cell proliferation rate was calculated as the ratio of (EdU+PDGFRA ${ }^{+}$ cells)/(PDGFRA ${ }^{+}$cells).

\section{Myofibroblast migration assay in vitro}

The migratory capacity of lung myofibroblasts was assessed by the Culture-Insert 2 Well system (ibidi) (32). Briefly, at postnatal (P) day 3, dissected lungs from control, Tfam flf; PdgfraCre/+and Mirolff; Pdgfra Crel+ mice were minced into small pieces and digested in solution $(1.2 \mathrm{U} / \mathrm{ml}$ dispase, $0.5 \mathrm{mg} / \mathrm{ml}$ collagenase $\mathrm{B}$ and $50 \mathrm{U} / \mathrm{ml}$ DNase I), rocking at $37^{\circ} \mathrm{C}$ for $2 \mathrm{hr}$ to release single cells. After adding an equal volume of culture medium (DMEM with 10\% FBS, 2x penicillin/streptomycin and 1x L-glutamine), the samples were filtered through $40 \mu \mathrm{m}$ cell strainers and centrifuged at $600 \mathrm{~g}$ for $10 \mathrm{~min}$. The dissociated cells were resuspended in $200 \mu \mathrm{l}$ of culture medium and plated into wells (100 $\mu$ l per well). The lung myofibroblasts were allowed to attach to the fibronectin-coated plates for 2-3 hr. Fresh culture medium was added and the attached myofibroblasts were cultured 2 or 3 days to reach $100 \%$ confluence. The confluent myofibroblasts were switched to starvation medium (DMEM with $0.5 \%$ FBS and 1x penicillin/streptomycin) for $16 \mathrm{hr}$ before removal of the insert. Myofibroblasts migration was assessed 36-48 hr afterwards. 


\section{Lentiviral production and transduction}

Lentiviruses were produced in HEK293T cells maintained in DMEM containing 10\% FBS, 1x penicillin/streptomycin and 1x L-glutamine (32). HEK293T cells were plated and transfected when they reached $70 \%$ confluence on the following day. $2 \mu \mathrm{g}$ of pMD2.G, $2 \mu \mathrm{g}$ of psPAX2, 4 $\mu \mathrm{g}$ of PDGFA-3xFLAG (in the modified pSECC lentiviral vector) and $50 \mu \mathrm{l}$ of polyethylenimine (PEI) $(1 \mu \mathrm{g} / \mu \mathrm{l})$ were mixed in $1000 \mu \mathrm{l}$ OPTI-MEM and added to a $10 \mathrm{~cm}$ dish. Incubation medium was replaced one day after transfection. $48 \mathrm{hr}$ post-transfection, the viral supernatant was collected, filtered through $0.45 \mu \mathrm{m}$ PVDF filter, then added to primary lung cells together with $8 \mu \mathrm{g} / \mathrm{ml}$ polybrene. $12 \mathrm{hr}$ post-transduction, the medium was replaced with fresh culture medium.

\section{PDGFA secretion assay}

PDGFA secretion assay was performed as previously described (32). In brief, PDGFA-3xFLAG was stably expressed in control and Tfam- and Mirol-deficient primary lung cells (derived from the lungs of control, Tfamflf; PdgfraCrel+ and Mirolflf; PdgfraCre/+ mice, respectively) that were plated onto $10 \mathrm{~cm}$ dishes. The culture media were replaced with OPTI-MEM supplemented with insulin, transferrin and selenium (ITS) once the cells reached $100 \%$ confluence. $24 \mathrm{hr}$ postincubation, the supernatants were mixed with $1 \mathrm{x}$ protein inhibitor cocktail, filtered through 0.45 $\mu \mathrm{m}$ filters, and centrifuged at high speed (>12000 rpm) for $15 \mathrm{~min}$ at $4^{\circ} \mathrm{C}$. The filtrates were then concentrated in protein concentration columns (Millipore CENTRICON YM-10 Centrifugal Filter Unit $2 \mathrm{~mL} 10 \mathrm{kDa}$ ) through centrifugation at $2000 \mathrm{~g}$ for $1 \mathrm{hr}$ at $4^{\circ} \mathrm{C}$. Concentrated supernatants were mixed with immunoprecipitation (IP) buffer (50 mM Tris pH 7.4, $2 \mathrm{mM}$ 
EDTA, $150 \mathrm{mM} \mathrm{NaCl}, 0.5 \%$ Triton X-100, 1x protein inhibitor cocktail) in a total volume of 500 $\mu$ l. Meanwhile, cells seeded on the plate were harvested and lysed in IP buffer.

Immunoprecipitation was performed using FLAG-M2 beads following the standard procedure. Western blotting was performed to detect PDGFA in the supernatants and lysates derived from control, Tfam-deficient, and Mirol-deficient primary lung cells.

\section{qPCR analysis}

qPCR was performed as previously described (32). Briefly, the right cranial lobe was dissected from the mouse lungs of the indicated genotypes and time points, and homogenized in $1 \mathrm{ml}$ TRIzol (Life Technologies). The homogenates were added to $200 \mu \mathrm{l}$ chloroform, and then centrifuged for $15 \mathrm{~min}$ at $12000 \mathrm{rpm}$. The upper aqueous layer was collected and mixed with an equal volume of $70 \%$ ethanol. RNAs were extracted with the RNeasy Mini Kit (Qiagen)

following the manufacturer's instructions. The extracted RNAs were reverse-transcribed with the Maxima First Strand cDNA Synthesis Kit (Thermo Scientific). Quantitative PCR (qPCR) was performed on the Applied Biosystems QuantStudio ${ }^{\text {TM }} 5$ Real-Time PCR System. Primers used for qPCR were: mouse Pdgfa forward, 5' -CTGGCTCGAAGTCAGATCCACA-3'; reverse, 5'GACTTGTCTCCAAGGCATCCTC-3', mouse Pdgfra forward, 5'-

TGCAGTTGCCTTACGACTCCAGAT-3'; reverse, 5'-

AGCCACCTTCATTACAGGTTGGGA-3', mouse Acta2 forward, 5'-

ATGCAGAAGGAGATCACAGC-3'; reverse, 5'-GAAGGTAGACAGCGAAGCC-3', mouse Eln forward, 5'-GCCAAAGCTGCCAAATACG-3'; reverse, 5' CTCCAGCTCCAACACCATAG-3', mouse Gapdh forward, 5'AGGTTGTCTCCTGCGACTTCA-3'; reverse, 5'-CCAGGAAATGAGCTTGACAAAGTT-3' . 


\section{Analysis of mtDNA/nDNA ratio}

Lung samples from Tfamflf; CAGGCreER/+, Rptorfff; $C A G G^{C r e E R /+}$ mice and human patients were lysed in lysis buffer (100 mM Tris pH 7.5, 5 mM EDTA, 0.4\% SDS, $200 \mathrm{mM} \mathrm{NaCl,} 50 \mu \mathrm{g} / \mathrm{ml}$

proteinase $\mathrm{K}$ ), and incubated in a $55^{\circ} \mathrm{C}$ chamber overnight. The tissues were digested with 100 $\mu \mathrm{g} / \mathrm{ml}$ RNase A at $37^{\circ} \mathrm{C}$ for 30 min to degrade the RNAs. The lysed samples were then added with equal volume of phenol/chloroform and centrifuged at 12,000 rpm for $10 \mathrm{~min}$. Lung DNAs were concentrated by ethanol precipitation. For mouse lungs, the mitochondrial 16S rRNA or ND1 gene and the nuclear $H k 2$ gene were used to calculate the relative ratio of mitochondrial (mt) to nuclear (n) DNA copy number. For human lungs, the mitochondrial tRNA ${ }^{\text {Leu(UUR) }}$ or $16 \mathrm{~S}$ rRNA gene and the nuclear $\beta 2$-microglobulin $(\beta 2 M)$ gene were employed to determine the relative ratio of mtDNA to nDNA. Quantitative PCR (qPCR) was performed on the Applied Biosystems QuantStudio $^{\text {TM }} 5$ Real-Time PCR System. The primer pairs used for the indicated genes were: mouse 16S rRNA forward, 5'-CCGCAAGGGAAAGATGAAAGAC-3'; reverse, 5'TCGTTTGGTTTCGGGGTTTC-3', mouse mt-ND1 forward, 5'-

CTAGCAGAAACAAACCGGGC-3'; reverse, 5'-CCGGCTGCGTATTCTACGTT-3', mouse Hk2 forward, 5'-GCCAGCCTCTCCTGATTTTAGTGT-3'; reverse, 5' -

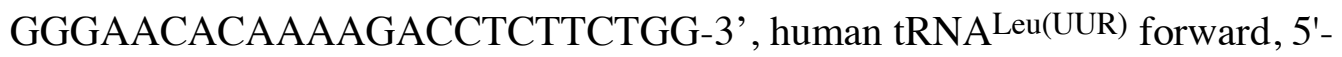
CACCCAAGAACAGGGTTTGT-3'; reverse, 5'-TGGCCATGGGTATGTTGTTA-3', human 16S rRNA forward, 5'-GCCTTCCCCCGTAAATGATA-3'; reverse, 5'TTATGCGATTACCGGGCTCT-3', human $\beta$-2-microglobulin $(\beta 2 M)$ forward, 5'TGCTGTCTCCATGTTTGATGTATCT-3'; reverse, 5'-TCTCTGCTCCCCACCTCTAAGT-3'. 


\section{Human lung tissues}

Human lung samples were processed as previously described (32). Briefly, lung tissues were obtained from severe Emphysema (Global Initiative for Chronic Obstructive Lung Disease Criteria, stages III or IV) at the time of lung transplantation. The donor control lung samples were indicated physiologically and pathologically normal (40). Written informed consent was obtained from all subjects and the study was approved by the University of California, San Francisco Institutional Review Board (IRB approval \# 13-10738).

\section{Western blotting analysis of human lung tissues}

Human lung tissues from normal subjects and emphysema patients were homogenized in RIPA buffer with 1x Protease Inhibitor Cocktail and 1x PMSF. The lysates were centrifuged with full speed for $15 \mathrm{~min}$ at $4{ }^{\circ} \mathrm{C}$ and analyzed by Western blotting as previously described (39). The primary antibodies used were: rabbit anti-TFAM (1: 2000, Proteintech, Cat\# 22586-1-AP), mouse anti-S6 Ribosomal Protein (1:2000, Cell Signaling Technology, Cat\# 2317), rabbit antiPhospho-S6 Ribosomal Protein (Ser235/236) (1:2000, Cell Signaling Technology, Cat\# 4856), mouse anti-alpha-tubulin (1:3000, Developmental Studies Hybridoma Bank, Cat\# 12G10).

\section{Statistical analysis}

All the statistical comparisons between different groups were shown as mean value \pm SEM. The P values were calculated by two-tailed Student's $t$-tests and statistical significance was evaluated as $(*) \mathrm{p}<0.05 ;(* *) \mathrm{p}<0.01 ;(* * *) \mathrm{p}<0.001$. More than or equal to three biological repeats were 
bioRxiv preprint doi: https://doi.org/10.1101/2021.04.09.439195; this version posted April 11, 2021. The copyright holder for this preprint (which

was not certified by peer review) is the author/funder, who has granted bioRxiv a license to display the preprint in perpetuity. It is made available under aCC-BY 4.0 International license.

performed, and the detailed biological replicates ( $\mathrm{n}$ numbers) were indicated in the figure legends. 


\section{Author contributions}

KZ, EY, and PTC conceived the project and designed the research. KZ, EY, JW and PTC

performed the experiments and analyzed the data. PW provided human lung tissues and analyzed the data. KZ, EY and PTC drafted the manuscript. All authors read and reviewed the manuscript.

\section{Acknowledgments}

Some data for this study were acquired at the Nikon Imaging Center at CVRI. This work was supported by R01 HL142876 from the National Institutes of Health to P.T.C. 


\section{References}

1. Burri PH. Structural aspects of postnatal lung development - alveolar formation and growth. Biol Neonate. 2006;89(4):313-22. doi:10.1159/000092868

2. Chao CM, Moiseenko A, Zimmer KP, Bellusci S. Alveologenesis: key cellular players and fibroblast growth factor 10 signaling. Mol Cell Pediatr. 2016;3(1):17. doi:10.1186/s40348016-0045-7

3. Whitsett JA, Weaver TE. Alveolar development and disease. Am J Respir Cell Mol Biol. 2015;53(1):1-7. doi:10.1165/rcmb.2015-0128PS

4. Silva DM, Nardiello C, Pozarska A, Morty RE. Recent advances in the mechanisms of lung alveolarization and the pathogenesis of bronchopulmonary dysplasia. Am J Physiol Lung Cell Mol Physiol. 2015;309(11):L1239-72. doi:10.1152/ajplung.00268.2015

5. Patel AR, Patel AR, Singh S, Singh S, Khawaja I. Global Initiative for Chronic Obstructive Lung Disease: The Changes Made. Cureus. 2019;11(6):e4985. doi:10.7759/cureus.4985

6. Barnes PJ, Burney PG, Silverman EK, Celli BR, Vestbo J, Wedzicha JA, et al. Chronic obstructive pulmonary disease. Nat Rev Dis Primers. 2015;1:15076. doi:10.1038/nrdp.2015.76 7. Rodriguez-Castillo JA, Perez DB, Ntokou A, Seeger W, Morty RE, Ahlbrecht K. Understanding alveolarization to induce lung regeneration. Respir Res. 2018;19(1):148. doi:10.1186/s12931-018-0837-5

8. Bostrom H, Willetts K, Pekny M, Leveen P, Lindahl P, Hedstrand H, et al. PDGF-A signaling is a critical event in lung alveolar myofibroblast development and alveogenesis. Cell. 1996;85(6):863-73. 
9. Lindahl P, Karlsson L, Hellstrom M, Gebre-Medhin S, Willetts K, Heath JK, et al.

Alveogenesis failure in PDGF-A-deficient mice is coupled to lack of distal spreading of alveolar smooth muscle cell progenitors during lung development. Development. 1997;124(20):3943-53.

10. Labbe K, Murley A, Nunnari J. Determinants and functions of mitochondrial behavior. Annu Rev Cell Dev Biol. 2014;30:357-91. doi:10.1146/annurev-cellbio-101011-155756

11. Chan DC. Mitochondrial Dynamics and Its Involvement in Disease. Annu Rev Pathol. 2019. doi:10.1146/annurev-pathmechdis-012419-032711

12. Devine MJ, Birsa N, Kittler JT. Miro sculpts mitochondrial dynamics in neuronal health and disease. Neurobiol Dis. 2016;90:27-34. doi:10.1016/j.nbd.2015.12.008

13. Laplante M, Sabatini DM. mTOR signaling in growth control and disease. Cell. 2012;149(2):274-93. doi:10.1016/j.cell.2012.03.017

14. Land SC, Scott CL, Walker D. mTOR signalling, embryogenesis and the control of lung development. Semin Cell Dev Biol. 2014;36:68-78. doi:10.1016/j.semcdb.2014.09.023

15. Varuzhanyan G, Rojansky R, Sweredoski MJ, Graham RL, Hess S, Ladinsky MS, et al. Mitochondrial fusion is required for spermatogonial differentiation and meiosis. Elife. 2019;8. doi:10.7554/eLife.51601

16. Muzumdar MD, Tasic B, Miyamichi K, Li L, Luo L. A global double-fluorescent Cre reporter mouse. Genesis. 2007;45(9):593-605. doi:10.1002/dvg.20335

17. Akiyama H, Kim JE, Nakashima K, Balmes G, Iwai N, Deng JM, et al. Osteochondroprogenitor cells are derived from Sox9 expressing precursors. Proc Natl Acad Sci U S A. 2005;102(41):14665-70. doi:10.1073/pnas.0504750102

18. Sun T, Jayatilake D, Afink GB, Ataliotis P, Nister M, Richardson WD, et al. A human YAC transgene rescues craniofacial and neural tube development in PDGFRalpha knockout 
mice and uncovers a role for PDGFRalpha in prenatal lung growth. Development. 2000;127(21):4519-29.

19. Gouveia L, Betsholtz C, Andrae J. Expression analysis of platelet-derived growth factor receptor alpha and its ligands in the developing mouse lung. Physiol Rep. 2017;5(6). doi:10.14814/phy2.13092

20. Bouda E, Stapon A, Garcia-Diaz M. Mechanisms of mammalian mitochondrial transcription. Protein Sci. 2019;28(9):1594-605. doi:10.1002/pro.3688

21. Hayashi S, McMahon AP. Efficient recombination in diverse tissues by a tamoxifeninducible form of Cre: a tool for temporally regulated gene activation/inactivation in the mouse. Dev Biol. 2002;244(2):305-18. doi:10.1006/dbio.2002.0597

22. Hamanaka RB, Glasauer A, Hoover P, Yang S, Blatt H, Mullen AR, et al. Mitochondrial reactive oxygen species promote epidermal differentiation and hair follicle development. Sci Signal. 2013;6(261):ra8. doi:10.1126/scisignal.2003638

23. Campbell H, Tomkeieff SI. Calculation of the internal surface of a lung. Nature. 1952;170(4316):116-7.

24. Escolar JD, Gallego B, Tejero C, Escolar MA. Changes occurring with increasing age in the rat lung: morphometrical study. Anat Rec. 1994;239(3):287-96. doi:10.1002/ar.1092390307 25. Venegas V, Halberg MC. Measurement of mitochondrial DNA copy number. Methods Mol Biol. 2012;837:327-35. doi:10.1007/978-1-61779-504-6_22

26. Nguyen TT, Oh SS, Weaver D, Lewandowska A, Maxfield D, Schuler MH, et al. Loss of Miro1-directed mitochondrial movement results in a novel murine model for neuron disease. Proc Natl Acad Sci U S A. 2014;111(35):E3631-40. doi:10.1073/pnas.1402449111 
27. Andrae J, Gouveia L, He L, Betsholtz C. Characterization of platelet-derived growth factor-A expression in mouse tissues using a lacZ knock-in approach. PLoS One. 2014;9(8):e105477. doi:10.1371/journal.pone.0105477

28. Roesch K, Jadhav AP, Trimarchi JM, Stadler MB, Roska B, Sun BB, et al. The transcriptome of retinal Muller glial cells. J Comp Neurol. 2008;509(2):225-38. doi:10.1002/cne.21730

29. Metzger RJ, Klein OD, Martin GR, Krasnow MA. The branching programme of mouse lung development. Nature. 2008;453(7196):745-50. doi:10.1038/nature07005

30. Yu K, Xu J, Liu Z, Sosic D, Shao J, Olson EN, et al. Conditional inactivation of FGF receptor 2 reveals an essential role for FGF signaling in the regulation of osteoblast function and bone growth. Development. 2003;130(13):3063-74.

31. Ruzzenente B, Metodiev MD, Wredenberg A, Bratic A, Park CB, Camara Y, et al. LRPPRC is necessary for polyadenylation and coordination of translation of mitochondrial mRNAs. EMBO J. 2012;31(2):443-56. doi:10.1038/emboj.2011.392

32. Zhang K, Yao E, Lin C, Chou YT, Wong J, Li J, et al. A mammalian Wnt5a-Ror2Vangl2 axis controls the cytoskeleton and confers cellular properties required for alveologenesis. Elife. 2020;9. doi:10.7554/eLife.53688

33. Sengupta S, Peterson TR, Laplante M, Oh S, Sabatini DM. mTORC1 controls fastinginduced ketogenesis and its modulation by ageing. Nature. 2010;468(7327):1100-4. doi:10.1038/nature09584

34. Shah M, Chacko LA, Joseph JP, Ananthanarayanan V. Mitochondrial dynamics, positioning and function mediated by cytoskeletal interactions. Cell Mol Life Sci. 2021(Online ahead of print.). 
35. Looney MR, Bhattacharya J. Live imaging of the lung. Annu Rev Physiol. 2014;76:43145. doi:10.1146/annurev-physiol-021113-170331

36. Gokerkucuk EB, Tramier M, Bertolin G. Imaging Mitochondrial Functions: from

Fluorescent Dyes to Genetically-Encoded Sensors. Genes (Basel). 2020;11(2).

doi:10.3390/genes11020125

37. Ding BS, Nolan DJ, Guo P, Babazadeh AO, Cao Z, Rosenwaks Z, et al. Endothelialderived angiocrine signals induce and sustain regenerative lung alveolarization. Cell. 2011;147(3):539-53. doi:10.1016/j.cell.2011.10.003

38. Swonger JM, Liu JS, Ivey MJ, Tallquist MD. Genetic tools for identifying and manipulating fibroblasts in the mouse. Differentiation. 2016;92(3):66-83. doi:10.1016/j.diff.2016.05.009

39. Lin C, Yao E, Zhang K, Jiang X, Croll S, Thompson-Peer K, et al. YAP is essential for mechanical force production and epithelial cell proliferation during lung branching morphogenesis. Elife. 2017;6. doi:10.7554/eLife.21130

40. Ware LB, Wang Y, Fang X, Warnock M, Sakuma T, Hall TS, et al. Assessment of lungs rejected for transplantation and implications for donor selection. Lancet. 2002;360(9333):619-20. doi:10.1016/s0140-6736(02)09774-X 


\section{Figure legends}

\section{Figure 1. Mitochondria display subcellular concentration in alveolar epithelial cells and}

\section{mesenchymal myofibroblasts of mouse lungs}

(A) Immunostaining of lung sections collected from Sox $9^{\mathrm{Cre} /+} ; \mathrm{ROSA} 26^{\mathrm{mTm} G /+}$ mice at 18.5 days post coitus $(\mathrm{dpc})$ and different postnatal (P) stages as indicated. The GFP signal identified alveolar epithelial cells while myofibroblasts were characterized by SMA expression. Moreover, mitochondria were labeled by MPC1; the trans-Golgi network was visualized by TGN38. Enhanced MPC1 signal was distributed non-uniformly in both alveolar epithelial cells and myofibroblasts. (B) Transmission electron micrographs of lungs collected from wild-type mice at P3. Prominent features in a given lung cell type include lamellar bodies in alveolar type II (AT2) cells, elongated cell membrane in alveolar type I (AT1) cells, and actin bundles and collagen fibers in myofibroblasts.

\section{Figure 2. Global inactivation of Tfam in postnatal mice results in alveolar defects}

(A) Schematic diagram of the time course of postnatal (P) administration of tamoxifen and harvest of mouse lungs. (B) Immunostaining of lungs collected from $C A G G^{C r e E R /+}$; ROSA26 $6^{m T m G /+}$ (control) and Tfamflf; $C A G G^{C r e E R /+} ; R O S A 26^{m T m G /+}$ mice at P10 that had received tamoxifen at P0. The GFP signal represents sites of induced CreER activity. Nuclear NKX2.1 staining marked all lung epithelial cells while PDGFRA immunoreactivity labeled mesenchymal myofibroblasts. (C) Hematoxylin and eosin-stained lung sections of control and Tfamflf; CAGGCreER/+ ; ROSA26 ${ }^{\mathrm{mTmG} /+}$ mice at P10. Histological analysis revealed the presence of enlarged saccules and retarded development of secondary septa in the mutant lungs. (D) Measurement of the mean linear intercept (MLI) in control and Tfamflf; $C A G G^{C r e E R /+}$; 
ROSA26 $6^{m T m G /+}$ lungs at $\mathrm{P} 10(\mathrm{n}=4$ for each group). The MLI was increased in Tfam-deficient lungs. (E) Immunostaining of lung sections collected from control and Tfamflf; CAGGCreER/+; ROSA26 $6^{\mathrm{mTm} /+}$ mice at P10. SMA expression was characteristic of myofibroblasts and phalloidin stained the actin filaments. (F) Quantification of the relative ratio of mitochondrial DNA (mtDNA), 16S rRNA and mtND1 (mitochondrially encoded NADH dehydrogenase 1), to nuclear DNA (nDNA), Hk2 (hexokinase 2), in lysates derived from control and Tfamflf; $C A G G^{C r e E R /+} ;$ ROSA26 $6^{m T m G /+}$ lungs (n = 4 for each group). (G) Immunostaining of lung sections collected from control and Tfamflf; $C A G G^{C r e E R /+} ; R O S A 26^{m T m G /+}$ mice at P10. MPC1 antibodies marked mitochondria; MTCO1 antibodies detected cytochrome c oxidase, the expression of which was controlled by Tfam. All values are mean SEM. $\left(^{*}\right) \mathrm{p}<0.05 ;(* *) \mathrm{p}<0.01$ (unpaired Student's $t$-test).

Figure 3. Elimination of Tfam or Mirol in the epithelium of mouse lungs disrupts alveolar

\section{formation}

(A) Hematoxylin and eosin-stained lung sections of control and Tfamflf; Sox9Cre/+ mice at different postnatal (P) stages as indicated. Histological analysis revealed the presence of enlarged saccules and failure in secondary septation in the mutant lungs. (B) Measurement of the MLI in control and Tfamflf; Sox9Crel+ lungs at P3-P10 ( $\mathrm{n}=3$ for each group). The MLI was increased in Tfam-deficient lungs. (C) Hematoxylin and eosin-stained lung sections of control and Mirolff; Sox $9 \mathrm{Cre} /+$ mice at different postnatal stages as indicated. Histological analysis detected enlarged saccules and lack of secondary septa in the mutant lungs. (D) Measurement of the MLI in control and Miroflf; Sox9Cre/+ lungs at P3-P10 ( $\mathrm{n}=3$ for each group). The MLI was increased in Mirol- 
deficient lungs. All values are mean SEM. $(* *) \mathrm{p}<0.01 ;(* * *) \mathrm{p}<0.001 ;$ ns, not significant (unpaired Student's $t$-test).

\section{Figure 4. Loss of epithelial Tfam or Mirol compromises PDGF release}

(A) Immunostaining of lungs collected from control and Tfamflf; Sox9Cre/+ mice at postnatal (P) day 5 or 10, some which were injected with EdU as indicated. (B) Immunostaining of lungs collected from control and Tfamflf; Sox9Crel+ mice at P5. (C) LacZ-staining (blue) of lung sections collected from Sox9Cre/+ $;$ Pdgfaex4COIN/+ (control) and Tfamflf; Sox9Crel+;

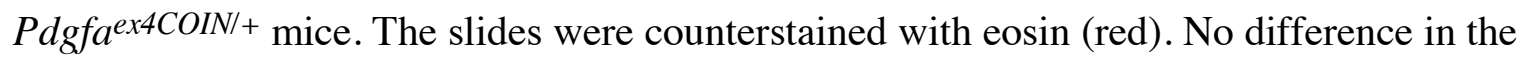
intensity of LacZ staining in the lung was noted between these two mouse lines. (D)

Quantification of myofibroblast proliferation in control and Tfam flf; Sox9Cre/+ lungs at P5, P7 and P10 ( $\mathrm{n}=3$ for each group). The rate of myofibroblast proliferation was calculated as the ratio of the number of $\mathrm{EdU}^{+}$myofibroblasts $\left(\mathrm{EdU}^{+} \mathrm{PDGFRA}^{+}\right)$to the number of myofibroblasts $\left(\right.$ PDGFRA $^{+}$). The percentage of proliferating myofibroblasts was reduced in Tfamflf; Sox 9 Cre/+ compared to controls at P7 and P10. (E) qPCR analysis of gene expression in control and Tfamflf; Sox9Cre/+ lungs at P5 ( $\mathrm{n}=3$ for each group). While no difference in expression levels was noted for Pdgfa and Pdgfra between control and Tfamflf; Sox 9 Cre/+ lungs, the expression levels of Acta2 (SMA) and Eln (elastin) were significantly reduced in the absence of Tfam. (F) Western blot analysis of cell lysates and supernatants from control and Tfam-deficient cells $(\mathrm{n}=4$ for each group) lentivirally transduced with PDGFA-expressing constructs. The amount of PDGFA released into the media was reduced in Tfam-deficient cells compared to controls. $\alpha$-Tubulin served as a loading control. (G) Immunostaining of lungs collected from control and Mirolfff; Sox9Cre/+ mice at P5, P7 or P10, some which were injected with EdU as indicated. $(\mathrm{H})$ 
Immunostaining of lungs collected from control and Mirolfff; Sox9Cre/+ mice at P5. (I)

Quantification of myofibroblast proliferation in control and Mirolfff; Sox9Cre/+ lungs at P7 and

P10 ( $n=3$ for each group). The percentage of proliferating myofibroblasts was reduced in

Mirolff; Sox9Cre/+ compared to controls at P7 and P10. (J) qPCR analysis of gene expression in

control and Mirolff; Sox9Cre/+ lungs at P5 ( $\mathrm{n}=3$ for each group). The expression levels of Pdgfa

and Pdgfra were unaltered between control and Mirolflf; Sox9Cre/+ lungs; the expression levels

of Acta2 and Eln were significantly reduced in the absence of Miro1. (K) Western blot analysis

of cell lysates and supernatants from control and Mirol-deficient cells ( $\mathrm{n}=4$ for each group)

lentivirally transduced with PDGFA-expressing constructs. The amount of PDGFA released into the media was reduced in Mirol-deficient cells compared to controls. $\alpha$-Tubulin served as a loading control. All values are mean SEM. $\left(^{*}\right) \mathrm{p}<0.05$; ns, not significant (unpaired Student's $t$ test).

Figure 5. Elimination of Tfam or Miro1 in mouse lung fibroblasts/myofibroblasts impairs alveolar formation

(A) Hematoxylin and eosin-stained lung sections of control and Tfamflf; PdgfraCre/+ mice at different postnatal (P) stages as indicated. Histological analysis revealed the presence of enlarged saccules and defective development of secondary septa in the mutant lungs. (B) Measurement of the MLI in control and Tfamflf; PdgfraCre/+ lungs at P0-P5 ( $\mathrm{n}=3$ for each group). The MLI was increased in Tfam-deficient lungs. (C) Hematoxylin and eosin-stained lung sections of control and Mirolfff; PdgfraCre/+ mice at different postnatal stages as indicated. Histological analysis detected enlarged saccules and lack of secondary septa in the mutant lungs. (D) Measurement of the MLI in control and Mirofff; PdgfraCre/+ lungs at P3-P10 ( $=3$ for each group). The MLI was 
increased in Mirol-deficient lungs. All values are mean SEM. $\left(^{*}\right) \mathrm{p}<0.05 ;\left({ }^{*}\right) \mathrm{p}<0.01$; ns, not significant (unpaired Student's $t$-test).

\section{Figure 6. Loss of mesenchymal Tfam or Miro1 compromises myofibroblast migration}

(A) Immunostaining of lungs collected from control and Tfamflf; PdgfraCre/+ mice at postnatal (P) day 2 and 5, some of which injected with EdU as indicated. (B) Quantification of myofibroblast proliferation in control and Tfamflf; PdgfraCre/+ lungs at P2, P3 and P5 (n = 3 for each group). The rate of myofibroblast proliferation was calculated as the ratio of the number of EdU $^{+}$myofibroblasts $\left(\right.$EdU $\left.^{+} \mathrm{PDGFRA}^{+}\right)$to the number of myofibroblasts $\left(\mathrm{PDGFRA}^{+}\right)$. The percentage of proliferating myofibroblasts was reduced in Tfamflf; PdgfraCrel+ compared to controls at P3 and P5. (C) qPCR analysis of gene expression in control and Tfamflf; PdgfraCre/+ lungs at P3 ( $\mathrm{n}=3$ for each group). The expression levels of Pdgfra, Acta2 and Eln were significantly reduced in Tfamflf; PdgfraCre/+ lungs compared to controls. (D) Wound recovery assays to assess the migratory ability of myofibroblasts derived from control and Tfamf/f; PdgfraCre/+ lungs ( $\mathrm{n}=3$ for each group). Within $36-48 \mathrm{hr}$, the wound area has been populated by migrating myofibroblasts derived from control lungs. By contrast, fewer myofibroblasts from Tfamflf; PdgfraCre/+ lungs reached the wound area within the same time frame. Wound recovery by myofibroblasts from control and Tfamflf; PdgfraCre/+ lungs was quantified. (E) Immunostaining of lungs collected from control and Mirolff; PdgfraCre/+ mice at P5 and P10, some of which were injected with EdU as indicated. (F) Quantification of myofibroblast proliferation in control and Tfamflf; $\mathrm{Pdgfra} C r e /+$ lungs at P5 and P10 (n = 3 for each group). The percentage of proliferating myofibroblasts was decreased in Mirolflf; PdgfraCre/+ compared to controls at P10. (G) qPCR analysis of gene expression in control and Mirolfff; PdgfraCrel+ lungs 
at P7 ( $\mathrm{n}=3$ for each group). The expression levels of Eln were significantly reduced in Mirolflf;

PdgfraCre/+ lungs in comparison with controls. (H) Wound recovery assays to assess the migratory ability of myofibroblasts derived from control and Mirolflf; PdgfraCre/+ lungs ( $\mathrm{n}=3$

for each group). Fewer myofibroblasts from Mirolfff; PdgfraCre/+ lungs reached the wound area within the same time frame compared to controls. Wound recovery by myofibroblasts from control and Mirolflf; PdgfraCre/+ lungs was quantified. All values are mean SEM. (*) p<0.05; ns, not significant (unpaired Student's $t$-test).

\section{Figure 7. Postnatal inactivation of Rptor in mice results in alveolar defects}

(A) Schematic diagram of the time course of postnatal (P) administration of tamoxifen and harvest of mouse lungs. (B) Hematoxylin and eosin-stained lung sections of control and Rptorfff; $C A G G^{C r e E R /+}$ mice at postnatal (P) day 10 . Histological analysis revealed the presence of enlarged saccules and retarded development of secondary septa in the mutant lungs. (C) Measurement of the mean linear intercept (MLI) in control and Rptorff; $C A G G^{C r e E R /+}$ lungs at P10 ( $\mathrm{n}=5$ for each group). The MLI was increased in Rptor-deficient lungs. (D) Immunostaining of lung sections collected from control and Rptorflf; $C A G G^{C r e E R /+}$; ROSA26 $6^{\mathrm{mTm} G /+}$ mice at P10. SMA expression was characteristic of myofibroblasts and phalloidin stained the actin filaments. (E). Quantification of the protein levels of ribosomal protein S6 (RPS6) and phosphorylated RPS6 (p-RPS6) in lung lysates derived from control and Rptorflf; $C A G G^{C r e E R /+} ;$ ROSA26 $6^{m T m G /+}$ lungs (n = 5 for each group). (F) Quantification of the relative ratio of mitochondrial DNA (mtDNA), 16S rRNA and mtND1 (mitochondrially encoded NADH dehydrogenase 1), to nuclear DNA (nDNA), Hk2 (hexokinase 2), in lysates derived from control and Rptorfff; CAGGCreER/+ $;$ ROSA26 $6^{m T m G /+}$ lungs (n = 5 for each group). (G) 
Immunostaining of lung sections collected from control and Rptorflf; $C A G G^{C r e E R /+}$;

ROSA26 $6^{\mathrm{mTm} /+}$ mice at P10. MPC1 antibodies marked mitochondria; MTCO1 antibodies

detected cytochrome c oxidase, the expression of which was controlled by Tfam. All values are mean SEM. $(*) \mathrm{p}<0.05 ;(* *) \mathrm{p}<0.01 ;(* * *) \mathrm{p}<0.001$ (unpaired Student's $t$-test).

Figure 8. Lungs from emphysema patients exhibit a reduction in mitochondrial DNA and

\section{TFAM expression}

(A) Hematoxylin and eosin-stained lung sections of normal and emphysema patients. Disruption

of alveoli in emphysema patients resulted in enlarged airspace with thin primary septa. (B) qPCR analysis of mitochondrial DNA (mtDNA) to nuclear DNA (nDNA) in lung lysates derived from normal and emphysema patients $\left(n=22\right.$ for each group). mtDNA-encoded tRNA ${ }^{\text {Leu }}$ and $16 \mathrm{~S}$ rRNA and nDNA-encoded $\beta 2 \mathrm{M}$ ( $\beta 2$ microglobulin) were used in this study. The relative ratio of mtDNA to nDNA was calculated. (C) Quantification of TFAM protein levels in lung lysates derived from normal and emphysema patients ( $n=21$ for each group). (D) Immunostaining of lungs collected from control and emphysema patients. Phalloidin detected actin filaments. (E). Quantification of the protein levels of ribosomal protein S6 (RPS6) and phosphorylated RPS6 (pRPS6) in lung lysates derived from normal and emphysema patients $(n=21$ for each group). All values are mean SEM. $(*) \mathrm{p}<0.05 ;(* *) \mathrm{p}<0.01$; ns, not significant (unpaired Student's $t$-test).

\section{Figure 9. A model of regulating alveolar formation through mitochondrial activity and}

\section{distribution}

The main players during alveolar formation are shown in the schematic diagram. Mitochondria display dynamic subcellular distribution in in alveolar epithelial cells and mesenchymal 
fibroblasts/myofibroblasts. Tfam controls mitochondrial activity while Mirol regulates mitochondrial distribution. Mitochondrial activity and distribution in alveolar epithelial cells (type I [AT1] and type II [AT2]) contribute to secretion of the PDGF-A ligand. Reception of PDGF-A by mesenchymal myofibroblasts is critical to myofibroblast proliferation and migration, a key step in secondary septation. Similarly, mitochondrial activity and distribution in myofibroblasts are also required for myofibroblast contraction and migration, likely through powering the cytoskeleton. The mTORC1 pathway plays a central role in controlling mitochondrial function during alveolar formation. Specification of alveolar epithelial cells and myofibroblasts was unaffected in mutant mouse lungs in which mitochondrial activity and distribution were perturbed. We propose that essential cellular processes have differential requirements of mitochondrial activity and distribution. Investigating how mitochondria control signaling pathways and cellular processes in vivo provide a new way to functionally define signaling pathways and cellular processes. We surmise that the regulatory circuitry mediated by mitochondria activity and distribution is also deployed during alveolar repair following lung injury. 

available under aCC-BY 4.0 International license.

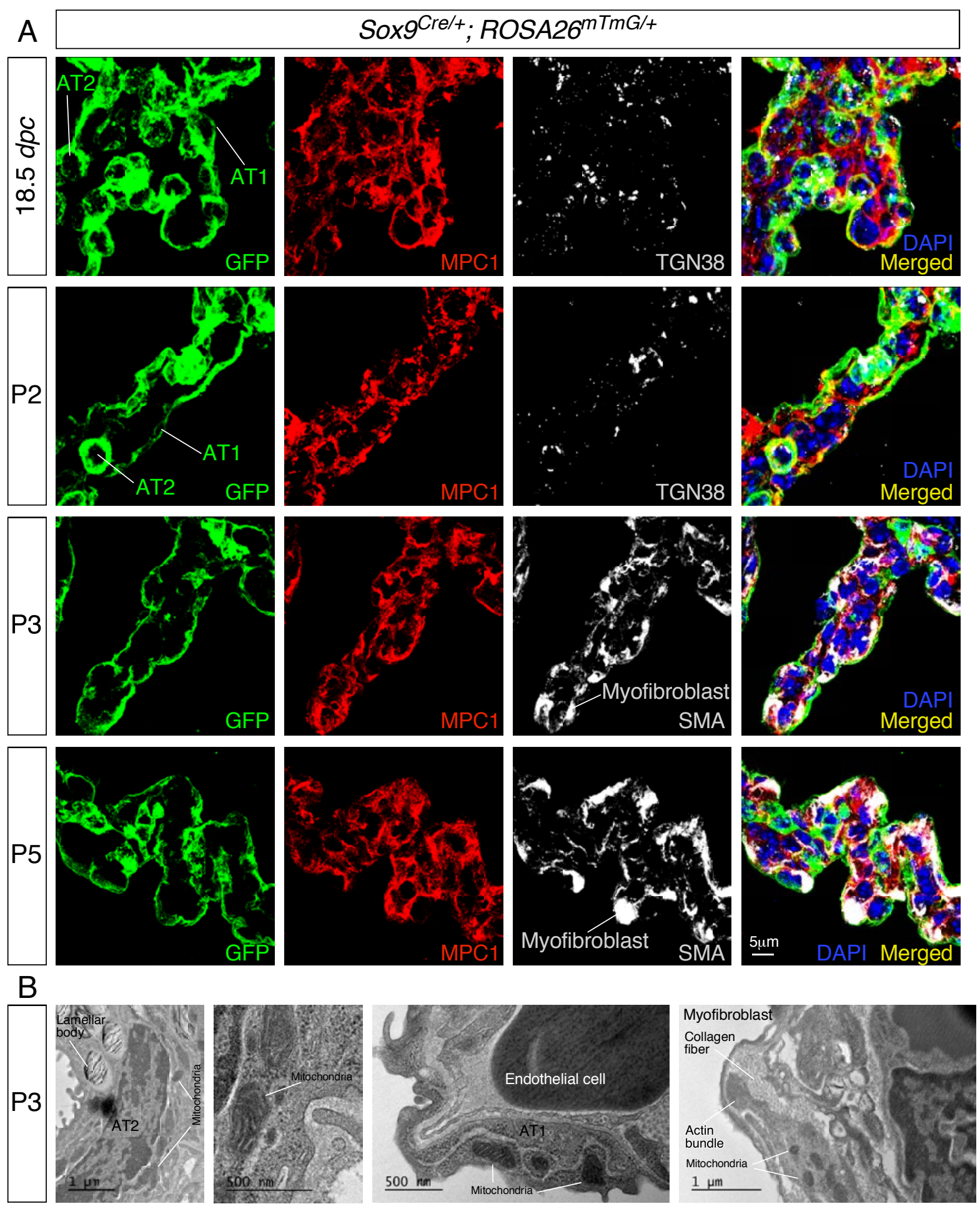




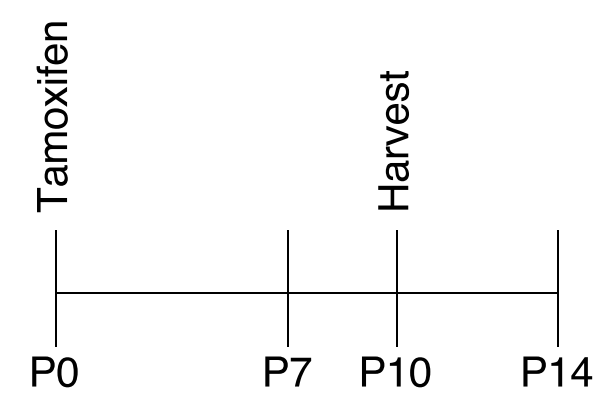

C

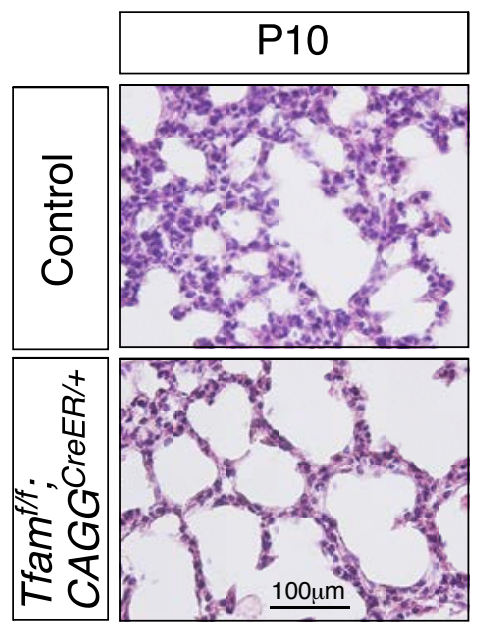

D
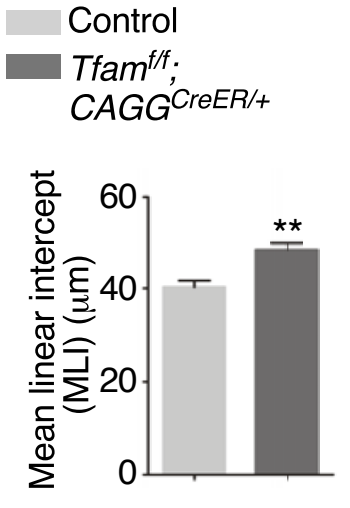

F

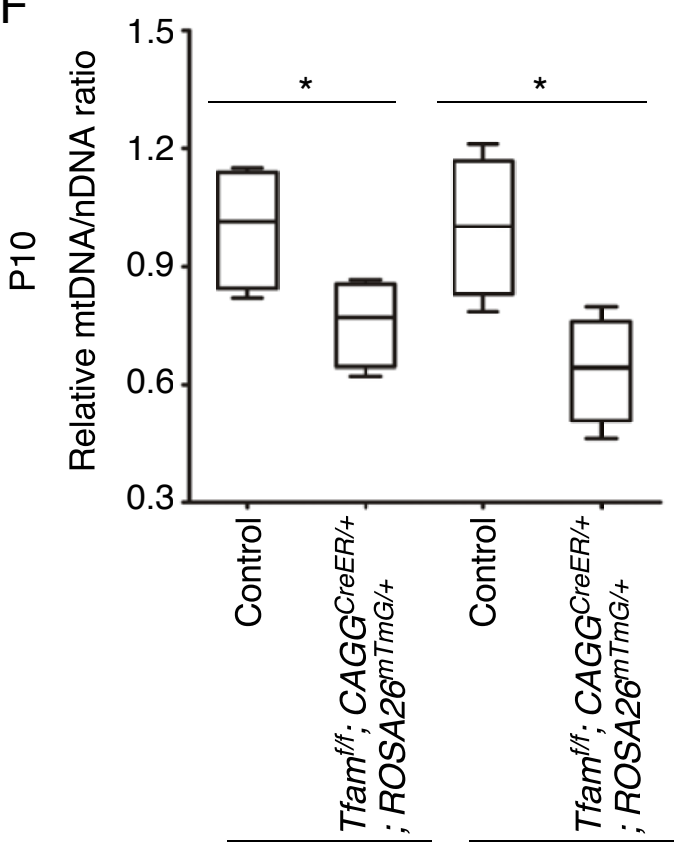

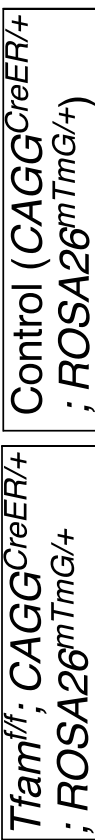

E
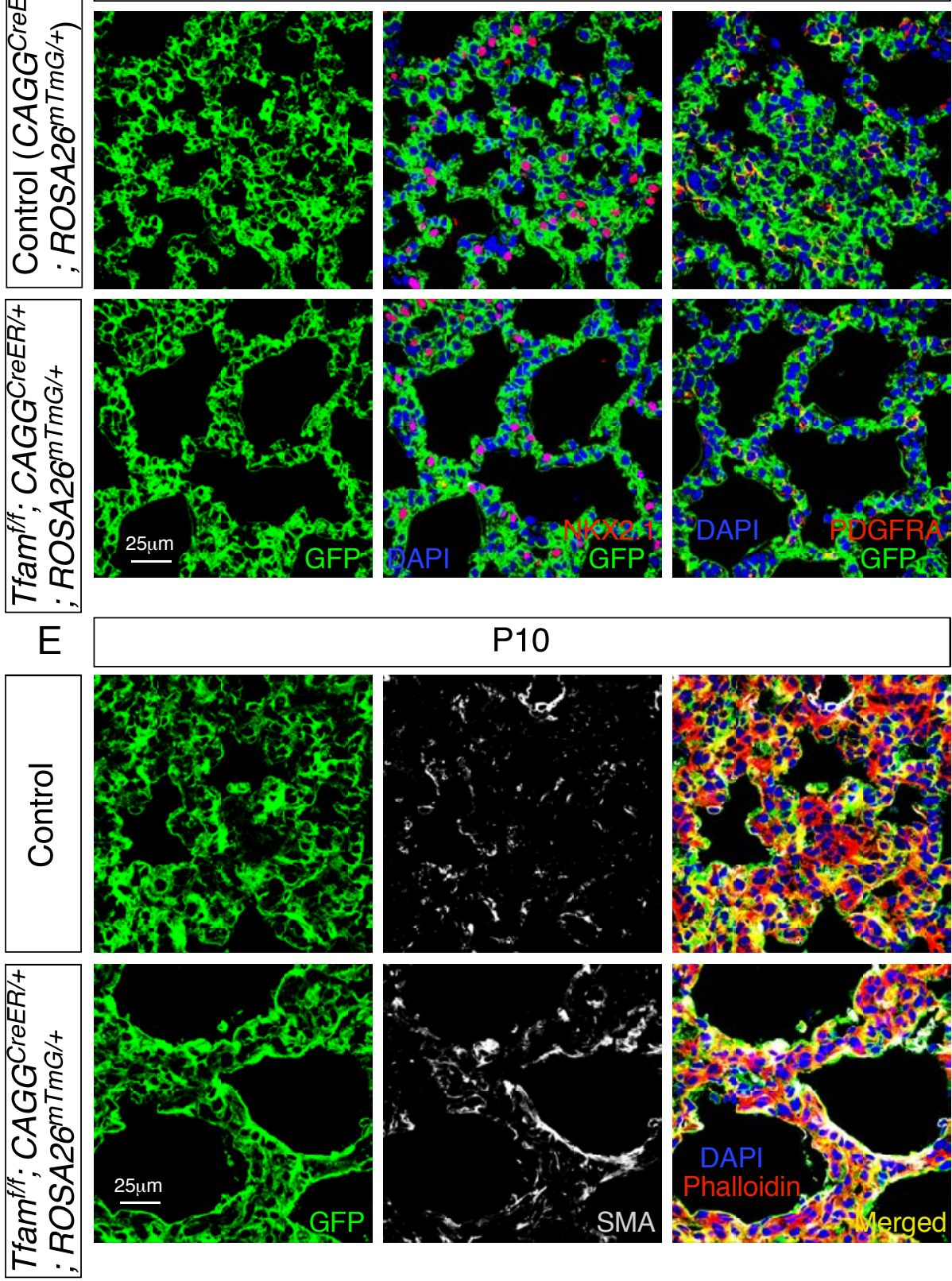

\section{P10}

G

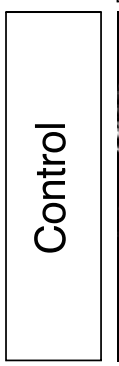

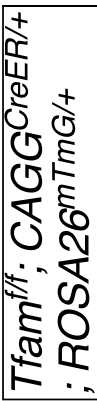
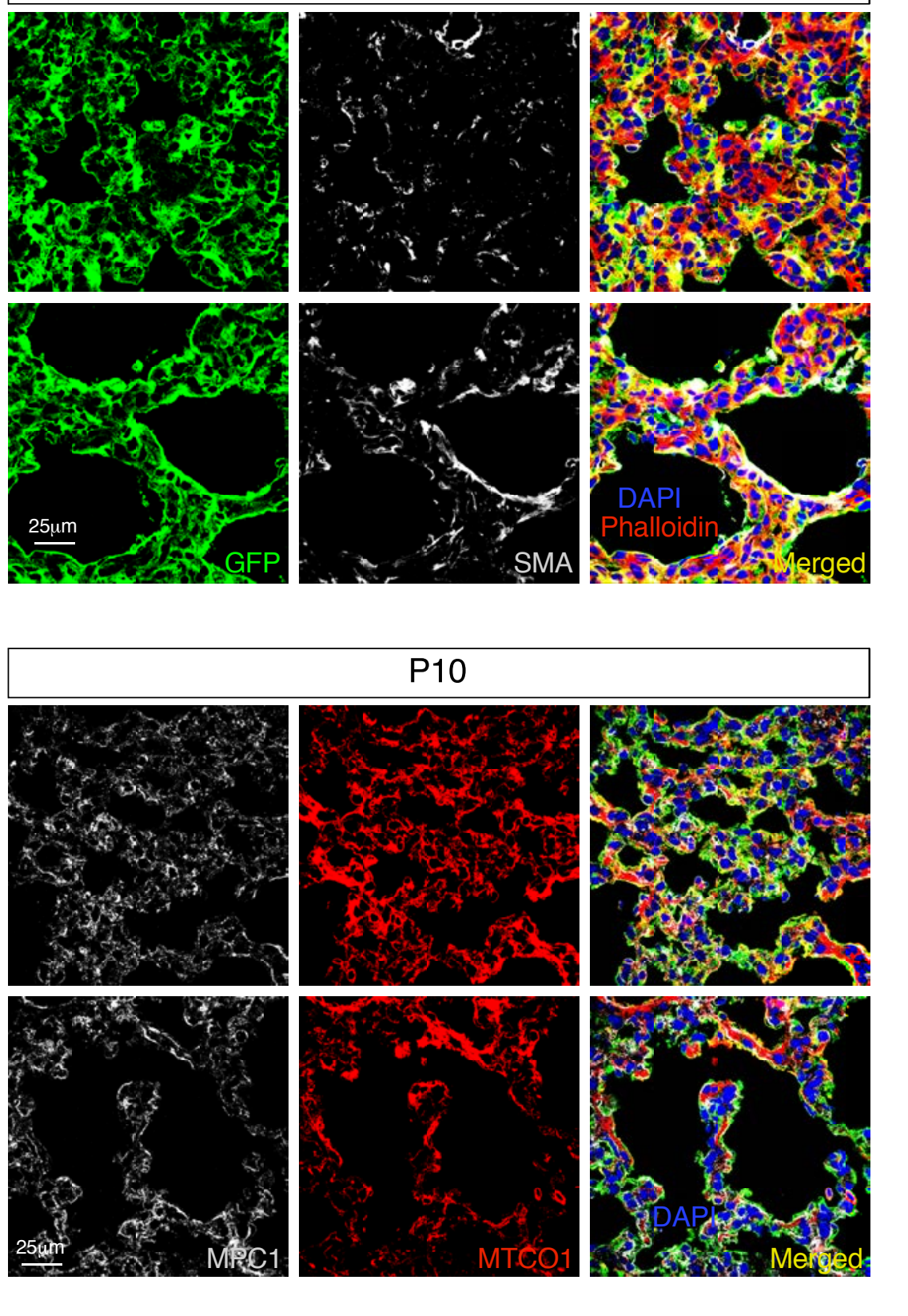
bioRxiv preprint doi: https://doi.org/10.1101/2021.04.09.439195; this version posted April 11, 2021. The copyright holder for this preprint (which Zhang etvaln available under aCC-BY 4.0 International license.
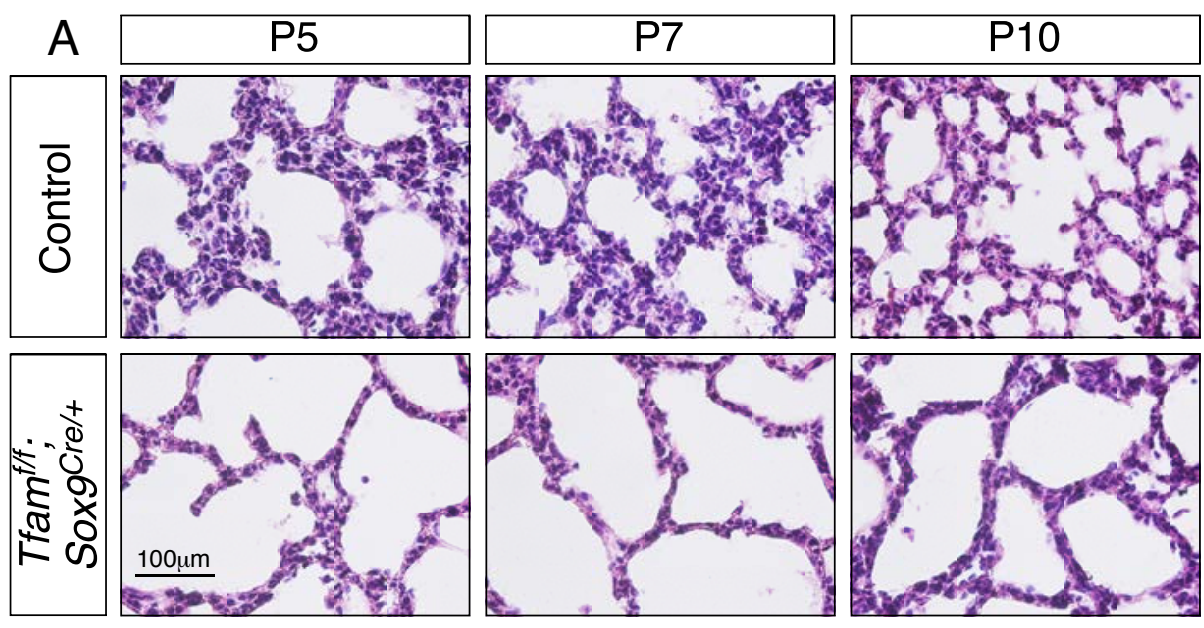

B
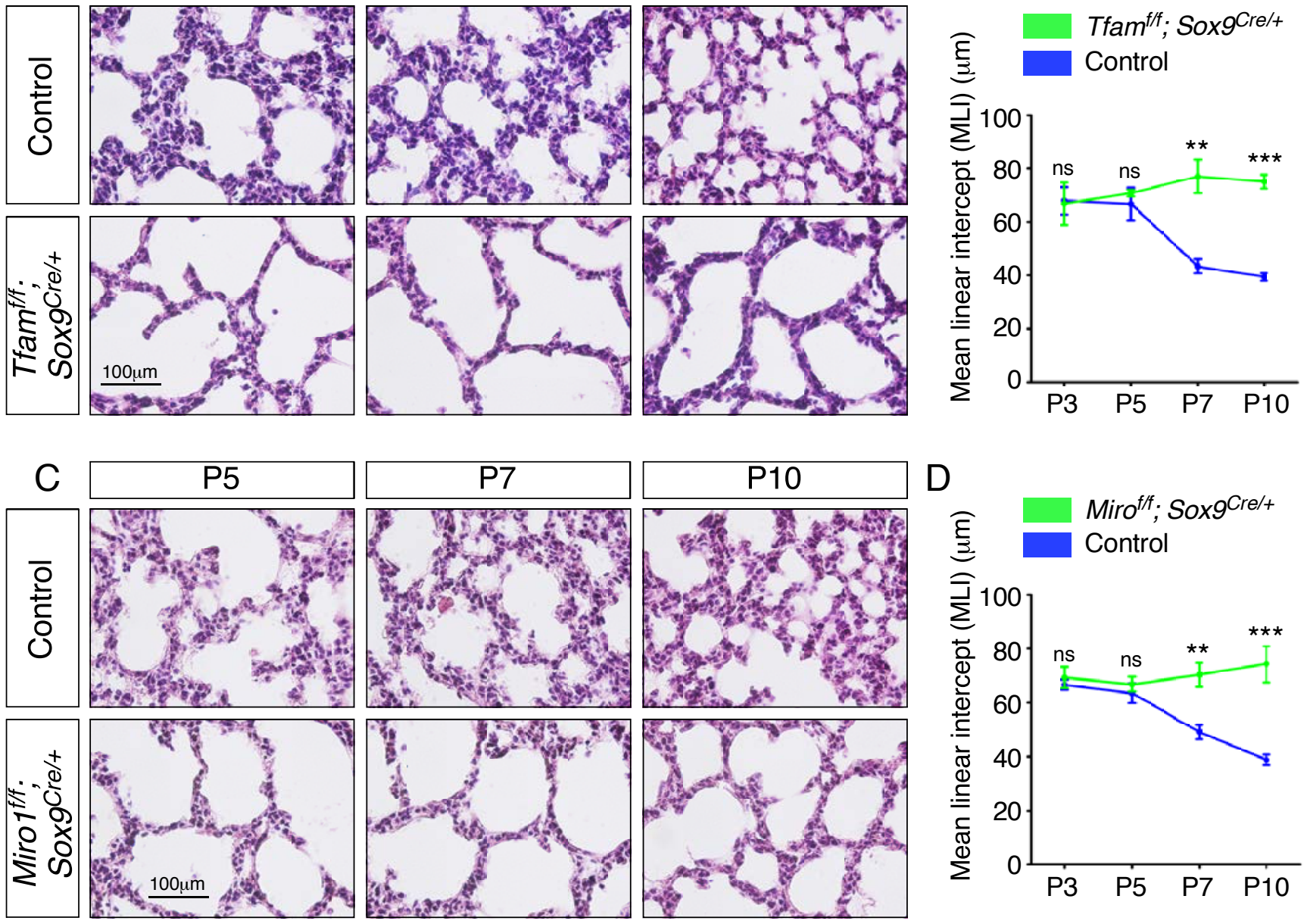
Zhang et al.bigigure 4 feprint doi: https://doi.org/10.1101/2021.04.09.439195; this version posted April 11.2021. The copyright holder for this preprint (which

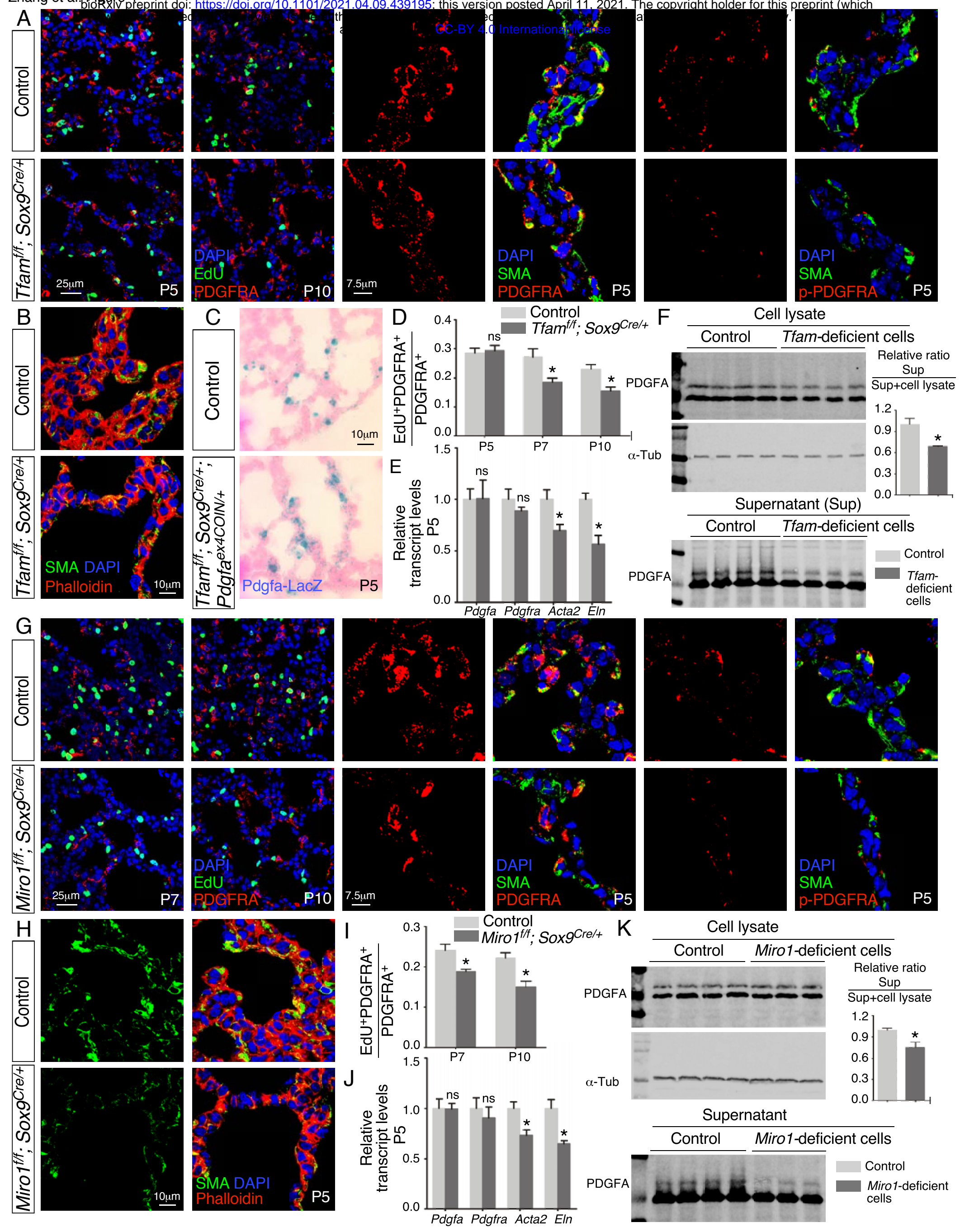



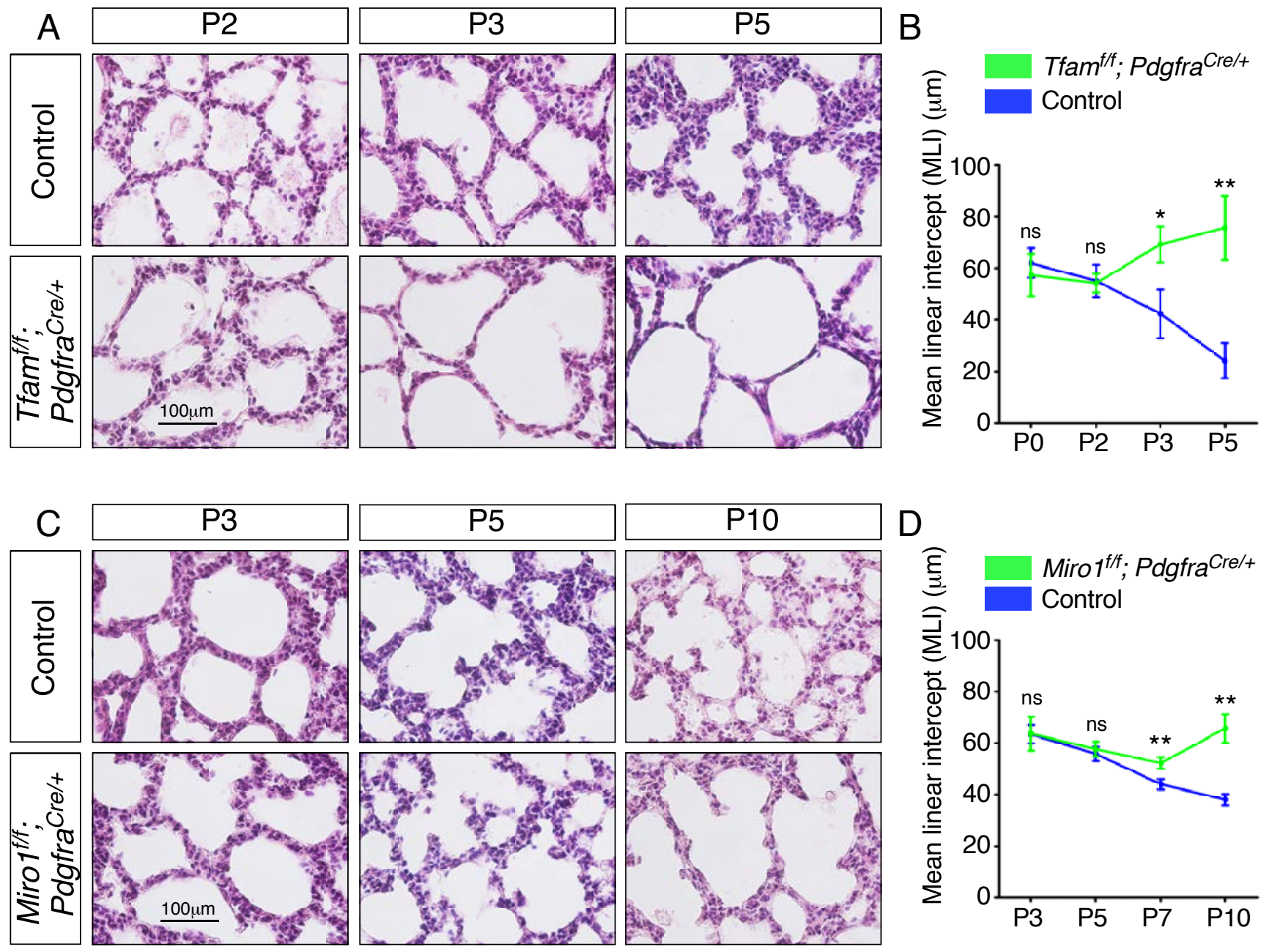


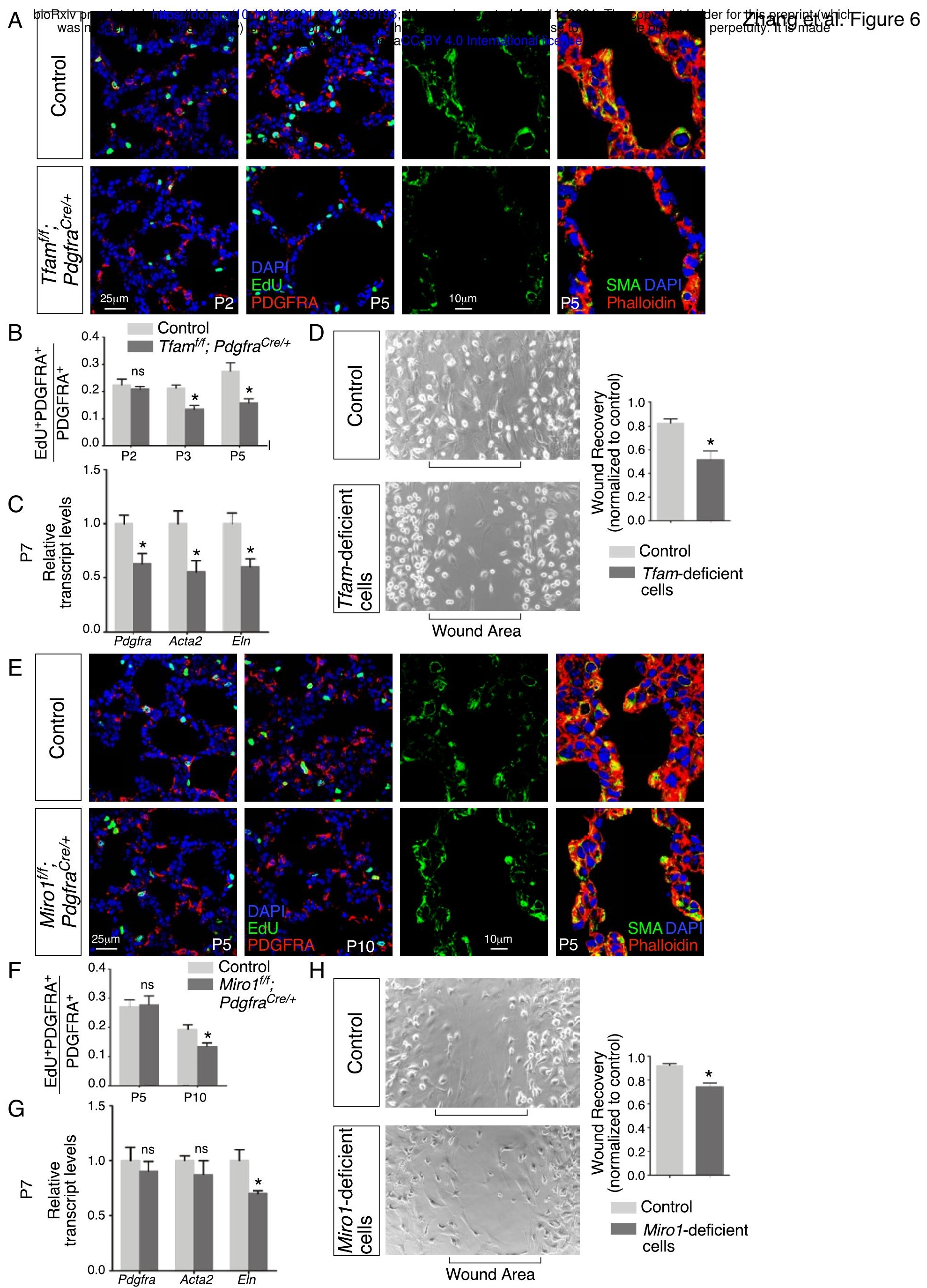


A
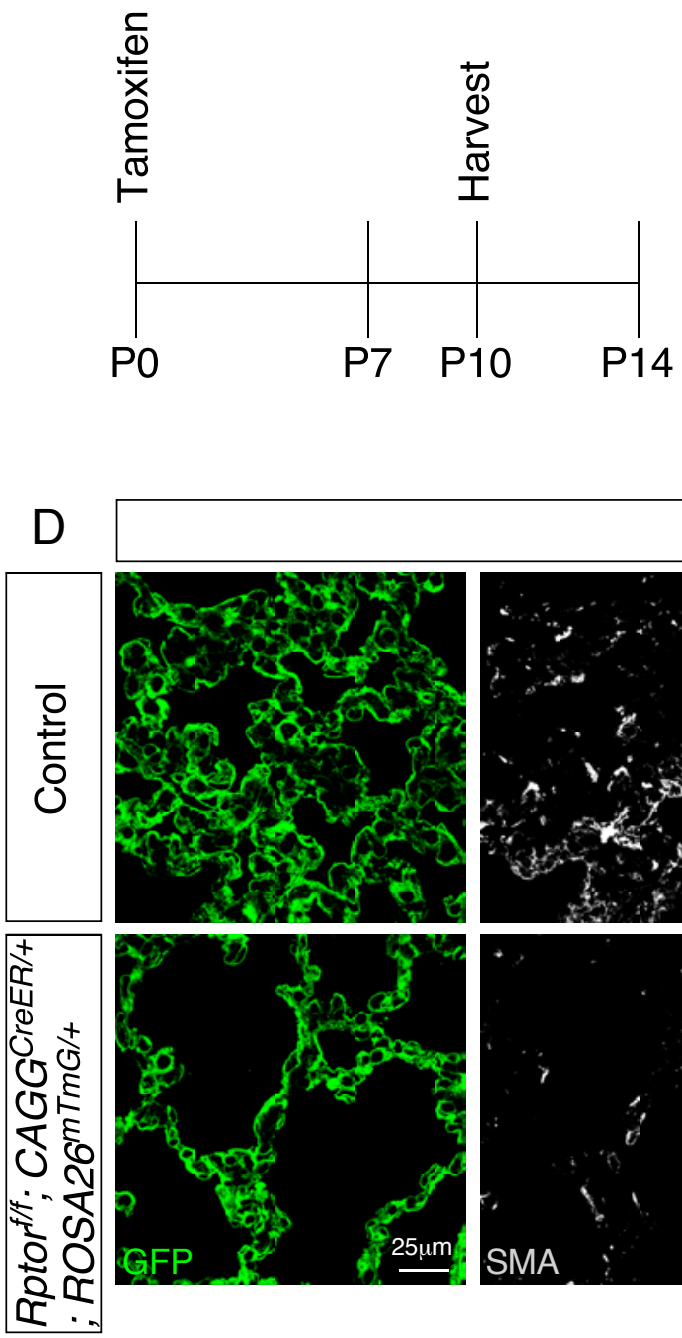

$\mathrm{F}$

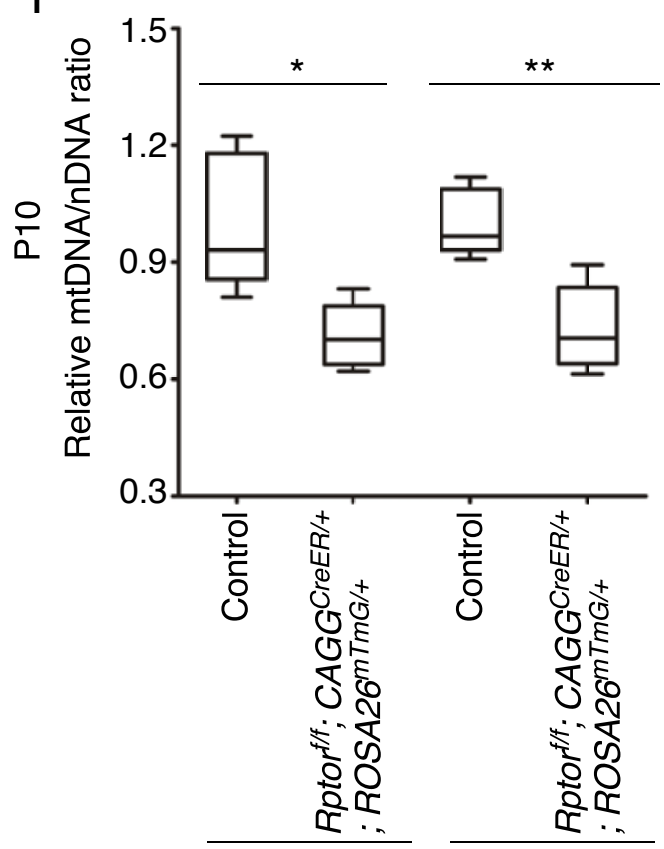

16S rRNA/Hk2 mtND1/Hk2

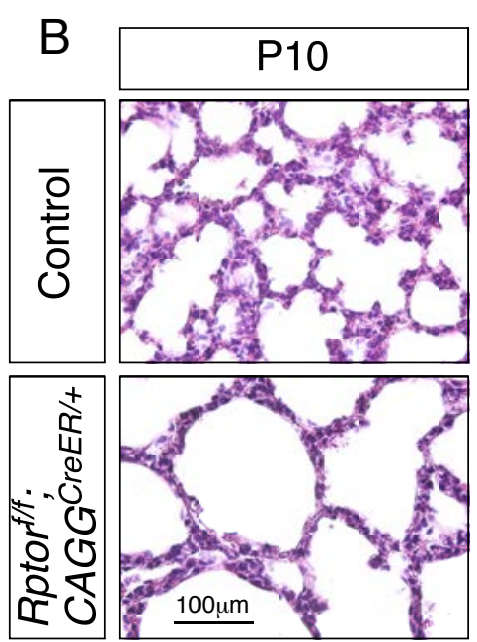

C

Control

Rptor ${ }^{f / f}$; CAGGCreER/+

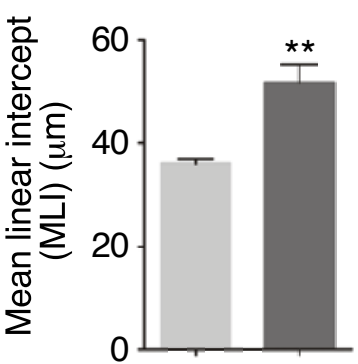

$E$

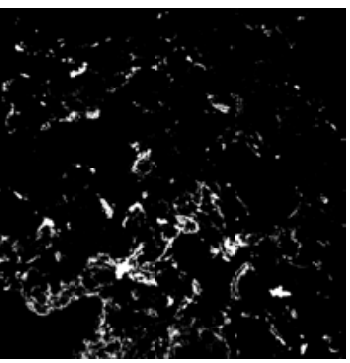

P10
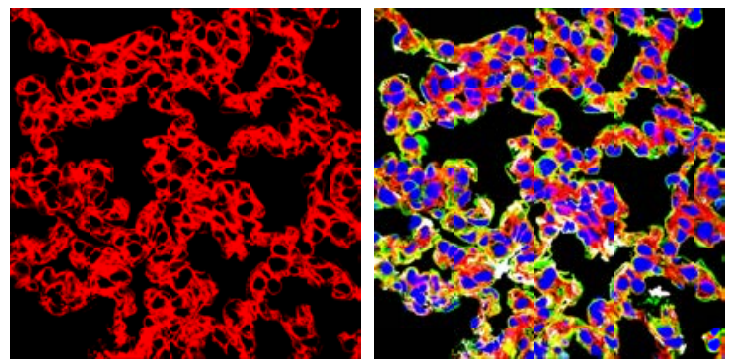

음
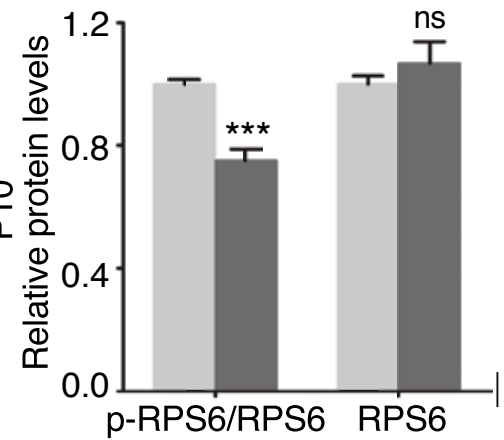

Control

Rptor $^{f / f} ;$ CAGGCreER/+
G
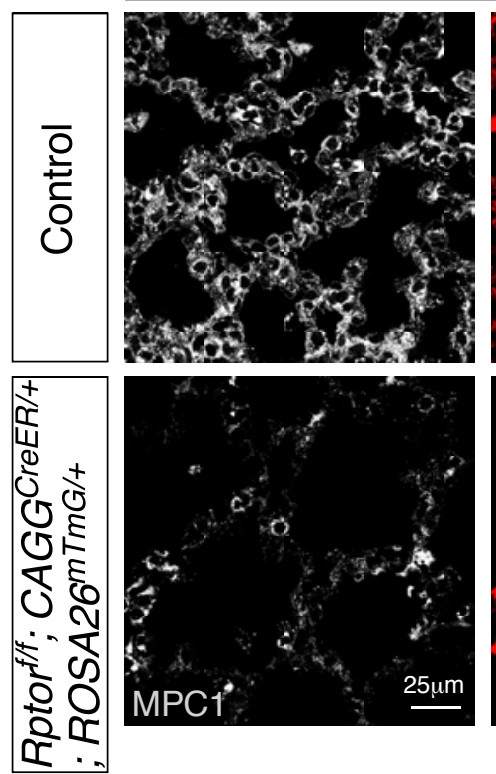

\section{P10}
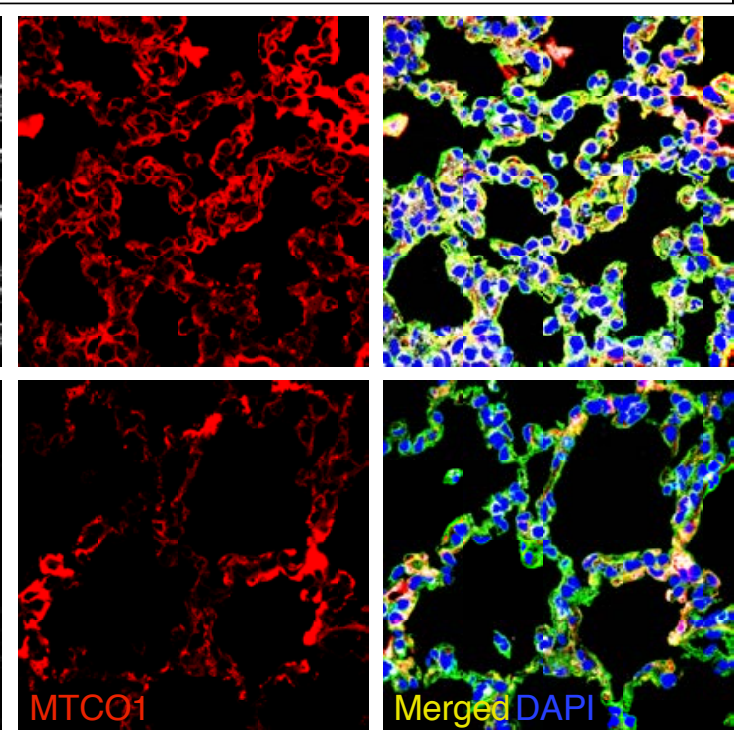
bioRxiv preprint doi: https://doi.org/10.1101/2021.04.09.439195; this version posted April 11, 2021. The copyright holder for this preprint (which Zhang et âl. neticertifieddy peer review) is the author/funder, who has granted bioRxiv a license to display the preprint in perpetuity. It is made
available under aCC-BY 4.0 International license.

A
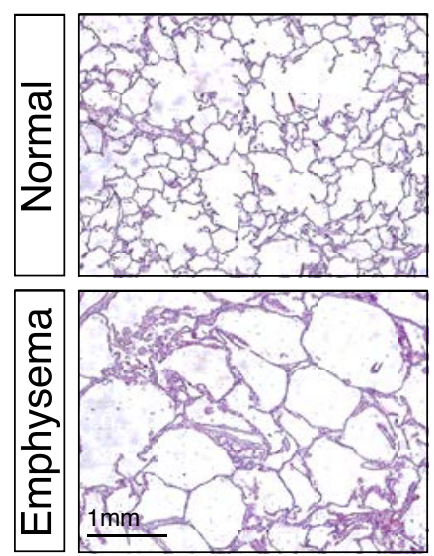

B

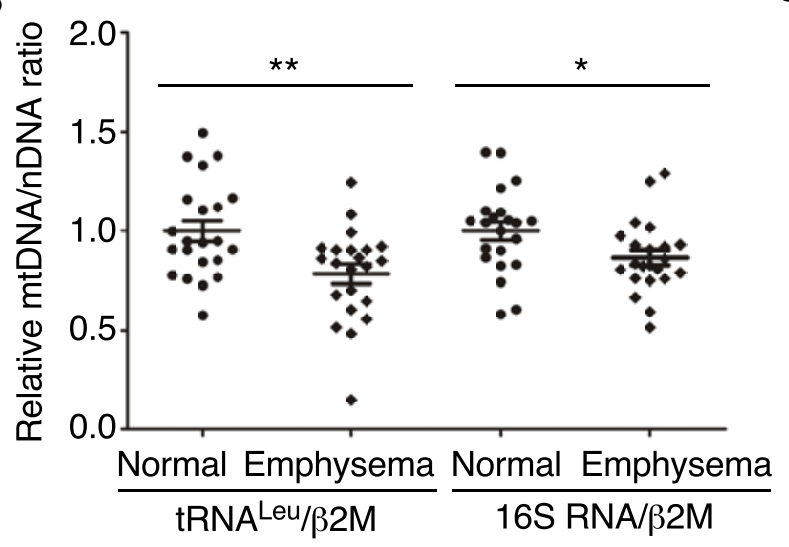

D
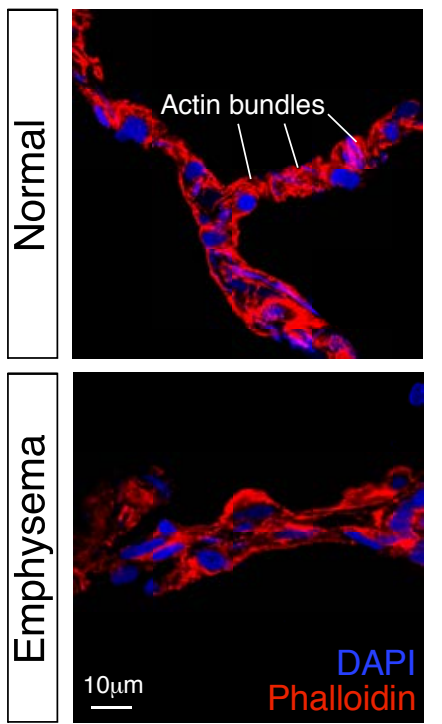

C
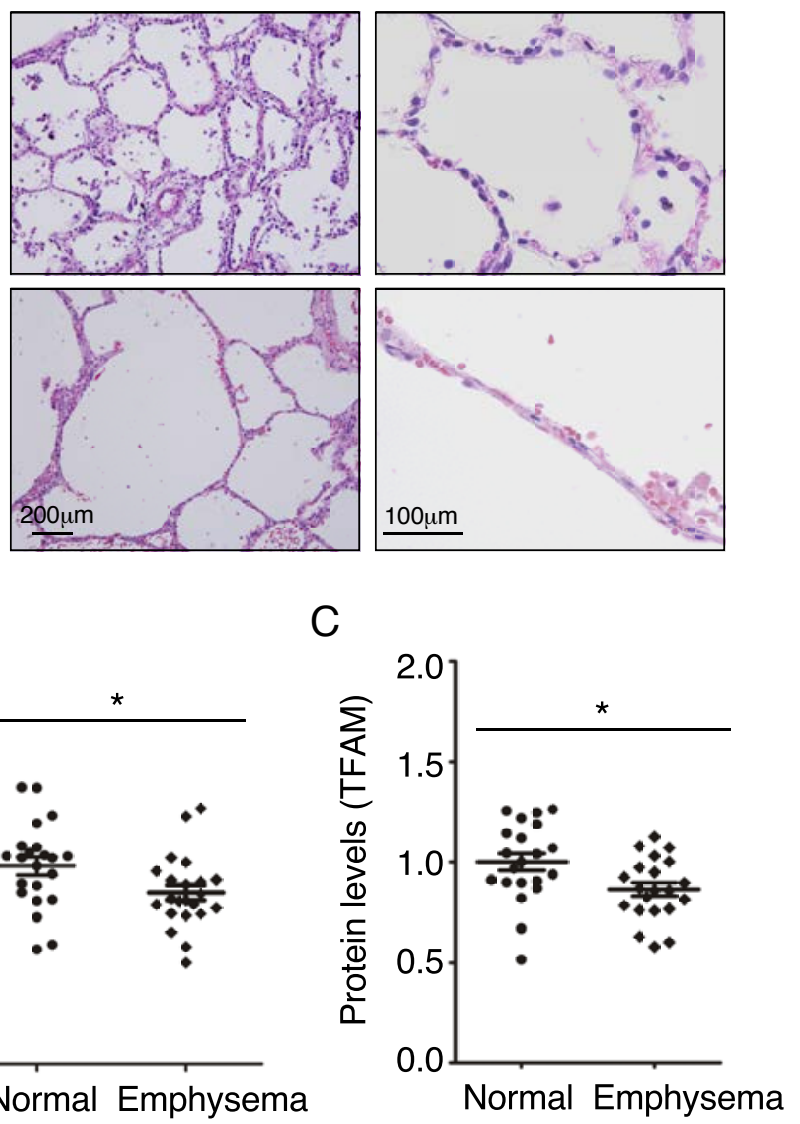

E
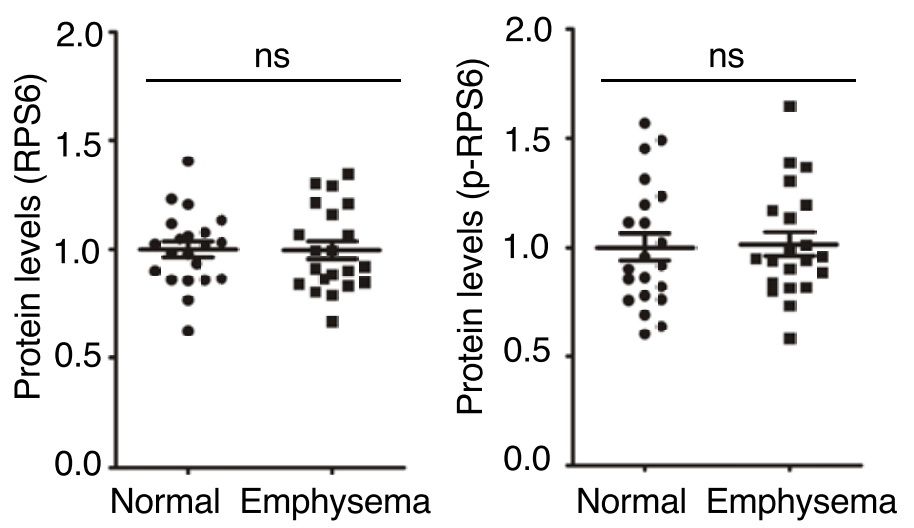


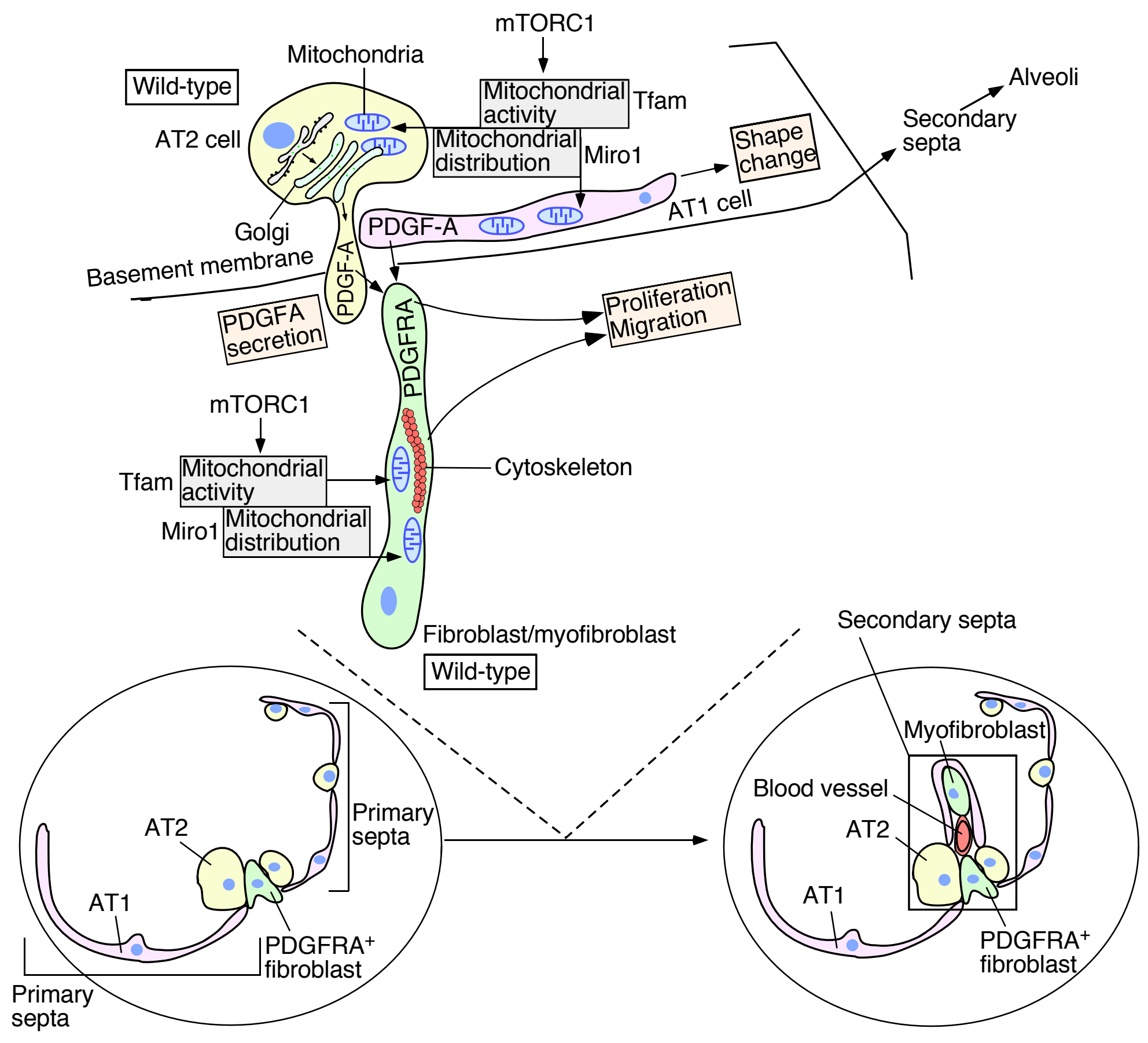



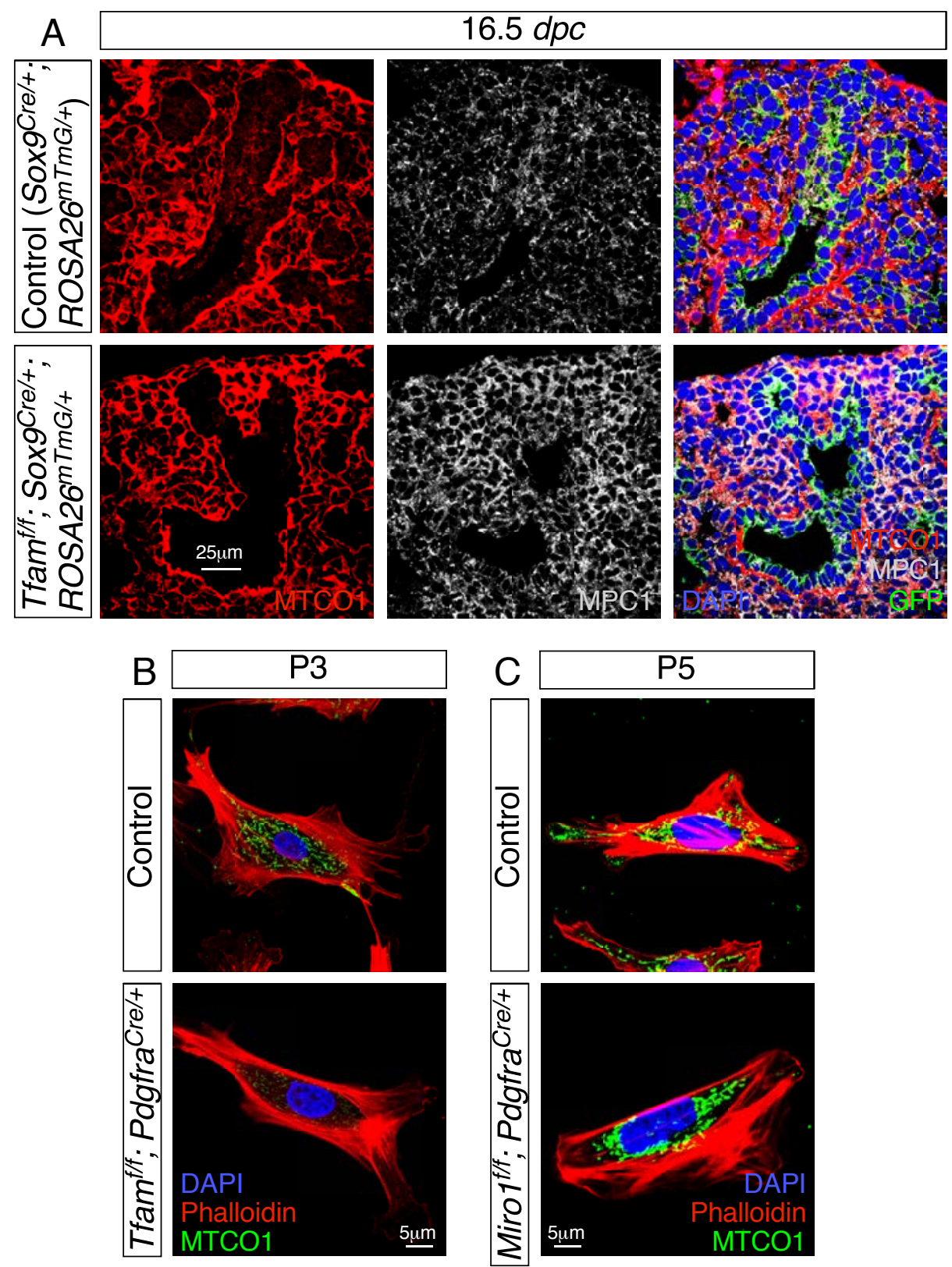

Figure 3-figure supplement 1. Loss Tfam or Miro1 disrupts mitochondrial activity and distribution, respectively

(A) Immunostaining of lung sections collected from Sox9Cre/+; ROSA26 ${ }^{\mathrm{mTmG} /+}$ (control) and Tfam $^{\text {f/f; }}$ Sox $9^{\mathrm{Cre} /+}$; ROSA26 $6^{\mathrm{mTmG} /+}$ mice at 16.5 days post coitus (dpc). MPC1 antibodies marked mitochondria while MTCO1 antibodies detected cytochrome $c$ oxidase, the expression of which was controlled by Tfam. (B) Immunostaining of fibroblasts derived from control or $\mathrm{Tfam}^{\mathrm{ff}}$; PdgfraCre/+ lungs at postnatal (P) day 3. (C) Immunostaining of fibroblasts derived from control or Miro1/ff; PdgfraCre/+ lungs at P5. 

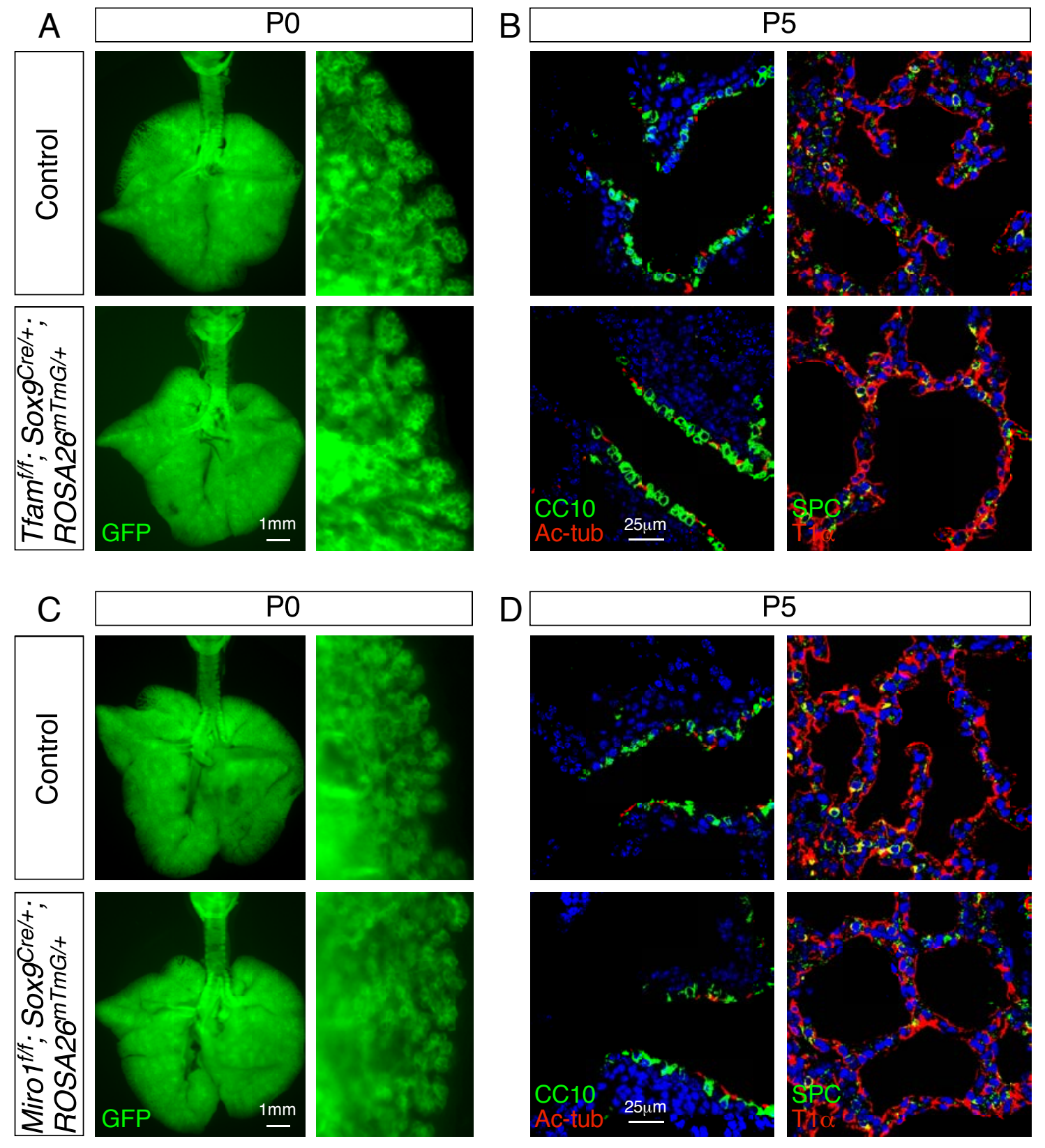

Figure 3-figure supplement 2. Elimination of Tfam or Miro1 in the epithelium of mouse lungs does not perturb saccule formation or cell type specification

(A) Surface view of dissected lungs from Sox $9^{C r e /+}$; ROSA26 $6^{m T m G /+}$ (control) and $T_{\text {fam }}^{\text {f/f; }}$; Sox9Cre/+; ROSA26 ${ }^{m T m G /+}$ mice at postnatal $(\mathrm{P})$ day 0 . No difference in saccule formation was noted between control and mutant lungs. (B) Immunostaining of lung sections from control and Tfam $^{f / f}$; SoxgCre/+; ROSA26 ${ }^{\mathrm{mTmG} /+}$ mice at P5. Specification of lung cell types (e.g., club cell

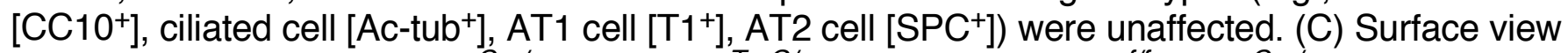

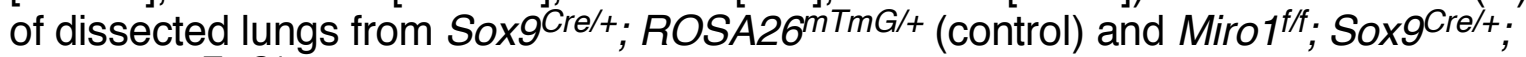
$R O S A 26^{m T m G /+}$ mice at postnatal $(P)$ day 0 . No difference in saccule formation was noted between control and mutant lungs. (D) Immunostaining of lung sections from control and Miroff; Sox9Cre/+; ROSA26 ${ }^{m T m G /+}$ mice at P5. Specification of lung cell types were unaffected. 

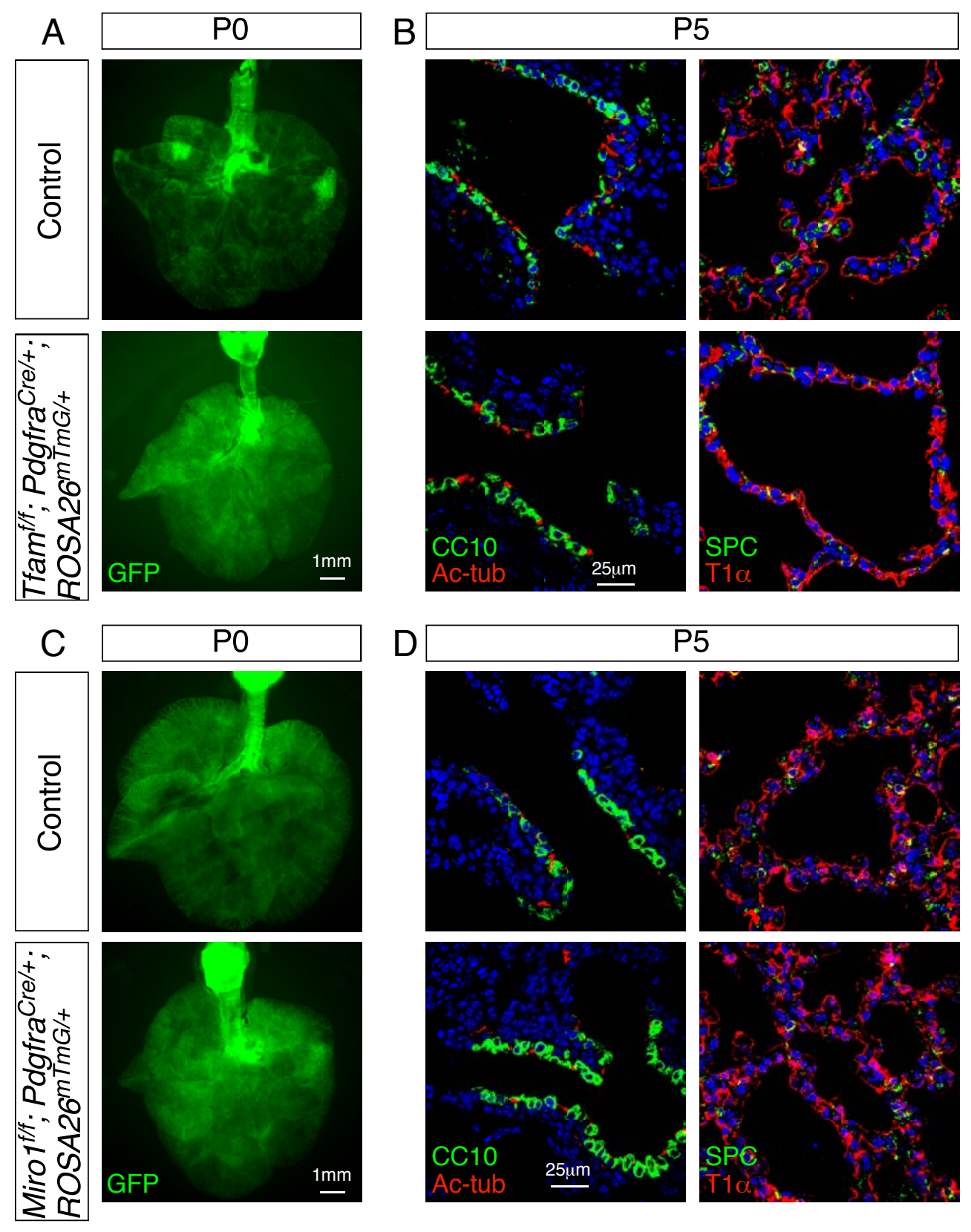

Figure 5-figure supplement 1. Removal of Tfam or Miro1 in mouse lung fibroblasts/myofibroblasts does not perturb saccule formation or cell type specification (A) Surface view of dissected lungs from PdgfraCre/+; ROSA26 ${ }^{\mathrm{mTmG} /+}$ (control) and $\mathrm{Tfam}^{\mathrm{ff} / \text {; }}$ PdgfraCre/+; ROSA26 ${ }^{m T m G /+}$ mice at postnatal $(\mathrm{P})$ day 0 . No difference in saccule formation was noted between control and mutant lungs. (B) Immunostaining of lung sections from control and Tfam $^{f / f}$; Pdgfra ${ }^{C r e /+} ;$ ROSA26 $6^{\mathrm{TmG} /+}$ mice at P5. Specification of lung cell types were unaffected. (C) Surface view of dissected lungs from control and Miro1f/f; Pdgfra Cre/+; ROSA26 ${ }^{\mathrm{mTmG} /+}$ mice at postnatal $(P)$ day 0 . No difference in saccule formation was noted between control and mutant lungs. (D) Immunostaining of lung sections from control and Miro ${ }^{f / f}$; PdgfraCre/t; ROSA26 ${ }^{\mathrm{mTmG} /+}$ mice at P5. Specification of lung cell types were unaffected. 

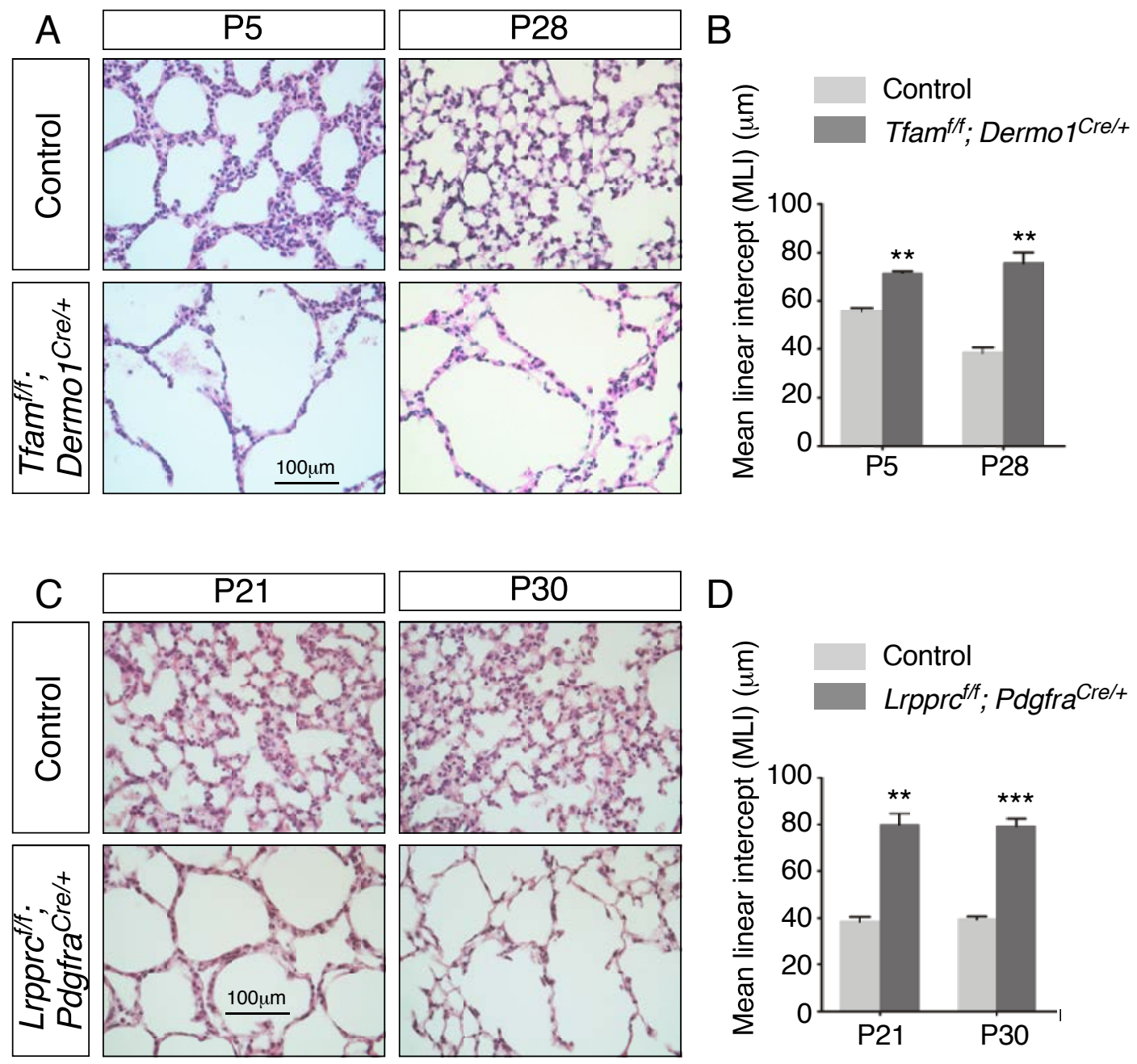

Figure 5-figure supplement 2. Inactivation of Tfam or Lrpprc in mouse lung fibroblasts/myofibroblasts leads to alveolar defects

(A) Hematoxylin and eosin-stained lung sections of control and $\mathrm{Tfam}^{\mathrm{f} / \mathrm{f}}$; Dermo1 ${ }^{\mathrm{Cre} /+}$ mice at different postnatal $(P)$ stages as indicated. Histological analysis revealed the presence of enlarged saccules and defective development of secondary septa in the mutant lungs. (B)

Measurement of the MLI in control and Tfam ${ }^{f / f}$; Dermo1 ${ }^{\text {Cre/t }}$ lungs at P5 and P28 ( $\mathrm{n}=3$ for each group). The MLI was increased in Tfam-deficient lungs. (C) Hematoxylin and eosin-stained lung sections of control and Lrpprc ${ }^{f / f}$; Pdgfra ${ }^{C r e /+}$ mice at P21 and P30. Saccules were enlarged with loss of secondary septation in the mutant lungs. (D) The MLI was increased in Lrpprc-deficient lungs at P21 and P30 ( $n=3$ for each group). All values are mean SEM. $\left(^{*}\right) p<0.05 ;\left(^{* *}\right) p<0.01$; $\left({ }^{* \star *}\right) p<0.001 ;$ ns, not significant (unpaired Student's t-test). 